\title{
JCMT POL-2 and BISTRO Survey Observations of Magnetic Fields in the L1689 Molecular Cloud
}

Kate Pattle $^{1,2}$ (D), Shih-Ping Lai ${ }^{2,3}$ (D) , James Di Francesco ${ }^{4,5}$ (D) , Sarah Sadavoy ${ }^{6}$ (D), Derek Ward-Thompson ${ }^{7}$ (D) , Doug Johnstone ${ }^{4,5}$ (iD), Thiem Hoang ${ }^{8,9}$ (D), Doris Arzoumanian ${ }^{10}$ (D), Pierre Bastien ${ }^{11}$ (D), Tyler L. Bourke ${ }^{12,13}$ (i), Simon Coudé ${ }^{14}$ (iD), Yasuo Doi ${ }^{15}$ (ID),

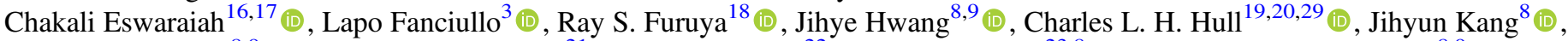
Kee-Tae Kim ${ }^{8,9}$ (D), Florian Kirchschlager ${ }^{21}$ (D), Jungmi Kwon ${ }^{22}$ (D), Woojin Kwon ${ }^{23,8}$ (i), Chang Won Lee (이창원) ${ }^{8,9}$ (iD), Tie Liu (刘铁) ${ }^{24}$ (D), Matt Redman ${ }^{1}$ (D), Archana Soam ${ }^{14}$ (D), Mehrnoosh Tahani ${ }^{25}$ (D), Motohide Tamura ${ }^{22,26,27}$ (D), and Xindi Tang ${ }^{28}$ (D) Centre for Astronomy, National University of Ireland Galway, University Road, Galway, Ireland; katherine.pattle@nuigalway.ie

${ }^{2}$ Institute for Astronomy and Department of Physics, National Tsing Hua University, No. 101, Section 2, Guangfu Road, Hsinchu 30013, Taiwan ${ }^{3}$ Academia Sinica Institute of Astronomy and Astrophysics, No. 1, Sec. 4, Roosevelt Road, Taipei 10617, Taiwan

${ }^{4}$ NRC Herzberg Astronomy and Astrophysics, 5071 West Saanich Road, Victoria, BC V9E 2E7, Canada

${ }^{5}$ Department of Physics and Astronomy, University of Victoria, Victoria, BC V8W 2Y2, Canada

${ }^{6}$ Department for Physics, Engineering Physics and Astrophysics, Queen's University, Kingston, ON K7L 3N6, Canada

${ }^{7}$ Jeremiah Horrocks Institute, University of Central Lancashire, Preston PR1 2HE, UK

${ }^{8}$ Korea Astronomy and Space Science Institute, 776 Daedeokdae-ro, Yuseong-gu, Daejeon 34055, Republic of Korea

${ }^{9}$ University of Science and Technology, Korea, 217 Gajeong-ro, Yuseong-gu, Daejeon 34113, Republic of Korea

${ }^{10}$ Instituto de Astrofísica e Ciências do Espaço, Universidade do Porto, CAUP, Rua das Estrelas, PT4150-762 Porto, Portugal

${ }^{11}$ Centre de recherche en astrophysique du Québec \& département de physique, Université de Montréal, C.P. 6128 Succ. Centre-ville, Montréal, QC H3C 3J7, Canada ${ }^{12}$ SKA Organisation, Jodrell Bank, Lower Withington, Macclesfield SK11 9FT, UK

13 Jodrell Bank Centre for Astrophysics, School of Physics and Astronomy, University of Manchester, Manchester M13 9PL, UK

${ }^{14}$ SOFIA Science Center, Universities Space Research Association, NASA Ames Research Center, Moffett Field, CA 94035, USA

15 Department of Earth Science and Astronomy, Graduate School of Arts and Sciences, The University of Tokyo, 3-8-1 Komaba, Meguro, Tokyo 153-8902, Japan

${ }^{16}$ CAS Key Laboratory of FAST, National Astronomical Observatories, Chinese Academy of Sciences, People's Republic of China

${ }^{17}$ National Astronomical Observatories, Chinese Academy of Sciences, A20 Datun Road, Chaoyang District, Beijing 100012, People's Republic of China

${ }^{18}$ Institute of Liberal Arts and Sciences Tokushima University, Minami Jousanajima-machi 1-1, Tokushima 770-8502, Japan

${ }^{19}$ National Astronomical Observatory of Japan, NAOJ Chile, Alonso de Córdova 3788, Office 61B, 7630422, Vitacura, Santiago, Chile

20 Joint ALMA Observatory, Alonso de Córdova 3107, Vitacura, Santiago, Chile

${ }^{21}$ Department of Physics and Astronomy, University College London, London WC1E 6BT, UK

22 Department of Astronomy, University of Tokyo, 7-3-1 Hongo, Bunkyo-ku, Tokyo 113-0033, Japan

${ }^{23}$ Department of Earth Science Education, Seoul National University, 1 Gwanak-ro, Gwanak-gu, Seoul 08826, Republic of Korea

${ }^{24}$ Key Laboratory for Research in Galaxies and Cosmology, Shanghai Astronomical Observatory, Chinese Academy of Sciences, 80 Nandan Road, Shanghai 200030, People's Republic of China

${ }^{25}$ Dominion Radio Astrophysical Observatory, Herzberg Astronomy and Astrophysics Research Centre, National Research Council Canada, P.O. Box 248, Penticton,

BC V2A 6J9, Canada
${ }^{26}$ Astrobiology Center, 2-21-1 Osawa, Mitaka-shi, Tokyo 181-8588, Japan

${ }^{27}$ National Astronomical Observatory, 2-21-1 Osawa, Mitaka-shi, Tokyo 181-8588, Japan

${ }^{28}$ Xinjiang Astronomical Observatory, Chinese Academy of Sciences, 830011 Urumqi, People's Republic of China Received 2020 July 10; revised 2020 October 23; accepted 2020 November 18; published 2021 February 1

\begin{abstract}
We present $850 \mu \mathrm{m}$ polarization observations of the L1689 molecular cloud, part of the nearby Ophiuchus molecular cloud complex, taken with the POL-2 polarimeter on the James Clerk Maxwell Telescope (JCMT). We observe three regions of L1689: the clump L1689N which houses the IRAS 16293-2433 protostellar system, the starless clump SMM-16, and the starless core L1689B. We use the Davis-Chandrasekhar-Fermi method to estimate plane-of-sky field strengths of $366 \pm 55 \mu \mathrm{G}$ in L1689N, $284 \pm 34 \mu \mathrm{G}$ in SMM-16, and $72 \pm 33 \mu \mathrm{G}$ in L1689B, for our fiducial value of dust opacity. These values indicate that all three regions are likely to be magnetically transcritical with sub-Alfvénic turbulence. In all three regions, the inferred mean magnetic field direction is approximately perpendicular to the local filament direction identified in Herschel Space Telescope observations. The core-scale field morphologies for L1689N and L1689B are consistent with the cloud-scale field morphology measured by the Planck Space Observatory, suggesting that material can flow freely from large to small scales for these sources. Based on these magnetic field measurements, we posit that accretion from the cloud onto L1689N and L1689B may be magnetically regulated. However, in SMM-16, the clump-scale field is nearly perpendicular to the field seen on cloud scales by Planck, suggesting that it may be unable to efficiently accrete further material from its surroundings.
\end{abstract}

Unified Astronomy Thesaurus concepts: Star formation (1569); Interstellar magnetic fields (845); Molecular clouds (1072); Interstellar medium (847); Polarimetry (1278); Submillimeter astronomy (1647); Star forming regions (1565)

\section{Introduction}

The role of magnetic fields in the process of star formation, and in particular the dynamic importance of magnetic fields in

${ }^{29}$ NAOJ Fellow. the later stages of the star formation process-the collapse of prestellar cores to form protostellar systems-remains poorly constrained. Magnetic fields are generally considered to act to resist the gravitational collapse of starless cores to form protostars (e.g., Mouschovias \& Spitzer 1976), although debate 
continues over whether magnetic fields mediate the star formation process or are subdominant or negligible in comparison to turbulence in the interstellar medium (ISM) (e.g., Hennebelle \& Inutsuka 2019; Krumholz \& Federrath 2019). In most ISM environments, dust grains are expected to be preferentially aligned, with their major axes perpendicular to the local magnetic field direction (Davis \& Greenstein 1951; Lazarian \& Hoang 2007; Andersson et al. 2015), and so dust emission polarimetry is a key tool for investigating magnetic fields in star-forming regions.

Magnetic fields in molecular clouds are preferentially aligned either parallel or perpendicular to dense elongated or filamentary structures (Soler et al. 2013; Planck Collaboration et al. 2016). These filaments are ubiquitous in star-forming regions, and may be the main site for the formation of Sun-like stars (Könyves et al. 2010). This has led to the suggestion that material flows onto filaments along magnetic field lines, until they have accreted sufficient mass to collapse under gravity to form a series of prestellar cores (Palmeirim et al. 2013; Soler et al. 2013; André et al. 2014).

The role of magnetic fields in the evolution of prestellar cores-gravitationally bound overdensities that will go on to form a single protostellar system (Ward-Thompson et al. 1994) -is not yet well characterized. A dynamically important magnetic field is broadly expected to support a prestellar core against, and to impose a preferred direction on, gravitational collapse (Mouschovias \& Spitzer 1976). Magnetically dominated systems might be expected to show the classical "hourglass" magnetic field indicative of gravitational collapse mediated by ambipolar diffusion (e.g., Fiedler \& Mouschovias 1993). Observations of the polarization geometry of prestellar cores typically show linear magnetic field geometries, oriented within $\sim 30^{\circ}$ of the minor axis of the core (Ward-Thompson et al. 2000; Kirk et al. 2006; Liu et al. 2019; Coudé et al. 2019). Hourglass fields have not been definitively observed in prestellar cores; however, such fields have been observed by interferometers in some protostellar sources, most famously in NGC 1333 IRAS 4A (Girart et al. 2006).

Recent interferometric observations of protostellar sources have shown that the majority have outflow direction uncorrelated with their overall magnetic field direction, while a minority may be strongly correlated (Hull et al. 2013). This has led to the suggestion that while magnetic fields are dynamically subdominant or even negligible in the majority of protostellar systems, there are a minority of magnetically dominated systems (Hull \& Zhang 2019).

In this work, we define clumps as subregions of a molecular cloud that contain sufficient mass to form multiple and distinct stellar systems. These stellar systems form from smaller substructures within the clumps, which we refer to as cores. Those substructures, which contain no protostellar sources, are referred to as starless cores, while those with embedded protostellar sources are referred to as protostellar cores. The gravitationally bound subset of starless cores we term prestellar cores. We note that cores need not be embedded in clumps; they can also exist within filamentary structures of the larger molecular cloud or in isolation.

In this work we present observations of three regions in the Ophiuchus L1689 molecular cloud with the POL-2 polarimeter on the James Clerk Maxwell Telescope (JCMT): the L1689N star-forming clump, the SMM-16 starless clump, and the L1689B starless core, all of which are embedded in a larger filamentary network. This paper is structured as follows: in Section 2 we briefly review the L1689 cloud and discuss its distance. In Section 3 we present our POL-2 observations of L1689. In Section 4 we estimate the magnetic field properties in L1689. In Section 5 we discuss grain alignment. In Section 6 we interpret our results. Section 7 summarizes this work.

\section{The L1689 Molecular Cloud}

The Ophiuchus molecular cloud is a nearby, well studied region of low-to-intermediate-mass star formation (Wilking et al. 2008), located at a distance $\sim 140 \mathrm{pc}$ from the solar system (Ortiz-León et al. 2018). The region is made up of two central dark clouds, L1688 and L1689 (Lynds 1962), both of which have extensive filamentary streamers to their NE, but are sharp-edged on their SW side (Vrba 1977). The strong asymmetry of the region is thought to be due to the influence of the nearby Sco OB2 association (Vrba 1977; Loren 1989), located to the west of and behind Ophiuchus (Mamajek 2008). The two main clouds and their filamentary streamers are shown in Figure 1, along with the central position of Sco OB2. Ophiuchus is threaded by a large-scale magnetic field, preferentially oriented $\sim 50^{\circ} \mathrm{E}$ of $\mathrm{N}$, as shown in Figure 1 (Vrba et al. 1976; Planck Collaboration et al. 2015; Kwon et al. 2015).

L1688 contains a number of dense clumps, including Oph A, $\mathrm{B}$, and $\mathrm{C}$, which were recently observed as part of the JCMT BISTRO (B-Fields in Star-forming Regions Observations) Survey (Ward-Thompson et al. 2017; Kwon et al. 2018; Soam et al. 2018; Liu et al. 2019). The Oph A region has also recently been observed in polarized far-infrared emission (Santos et al. 2019). These observations have shown that the dense clumps within L1688 have a mean magnetic field direction consistent with the large-scale NE/SW field, but with significant deviations, both ordered and disordered, from the mean field direction.

L1688 contains more than 20 embedded protostars and two B stars, whereas L1689 contains only five embedded protostellar systems (Enoch et al. 2009). Nutter et al. (2006) proposed that there is a star formation gradient across Ophiuchus driven by the global influence from the Sco OB2 association (Loren 1989), under the assumption that L1689 is located further from Sco OB2 than L1688. However, the evolution of dense gas within the two clouds also appears to be strongly influenced by local effects, without clear evidence for a W-E star formation gradient within L1688 (Pattle et al. 2015).

Although L1688 (R.A., decl. $=16^{\mathrm{h}} 28^{\mathrm{m}},-24^{\circ} 32^{\prime} ; l, b=$ $353.22,16^{\circ} .53$ ) and L1689 (R.A., decl. $=16^{\mathrm{h}} 32^{\mathrm{m}},-24^{\circ} 28^{\prime}$; $\left.l, b=353.94,15^{\circ} .84\right)$ have previously been treated as being at the same distance, revised distance estimates, combining Gaia DR2 and Very Long Baseline Array parallaxes, place L1689 5.8 pc behind L1688, with the two clouds located at distances of $144.2 \pm 1.3 \mathrm{pc}$ and $138.4 \pm 2.6 \mathrm{pc}$ respectively (Ortiz-León et al. 2018). The Sco OB2 association is located behind the Ophiuchus molecular cloud, at R.A., decl. = $16^{\mathrm{h}} 11^{\mathrm{m}},-23^{\circ} 18^{\prime}\left(l, b=351^{\circ} .5,20^{\circ} .2\right)$ and at a distance of $145 \pm 2$ pc (de Zeeuw et al. 1999). The plane-of-sky and lineof-sight locations of the clouds and of Sco OB2 are shown in Figure 1. Coordinates for each object are taken from the Simbad database (Wenger et al. 2000). We note that these revised distance estimates suggest that L1688 and L1689 are located at similar distances to Sco OB2, with the distance from 


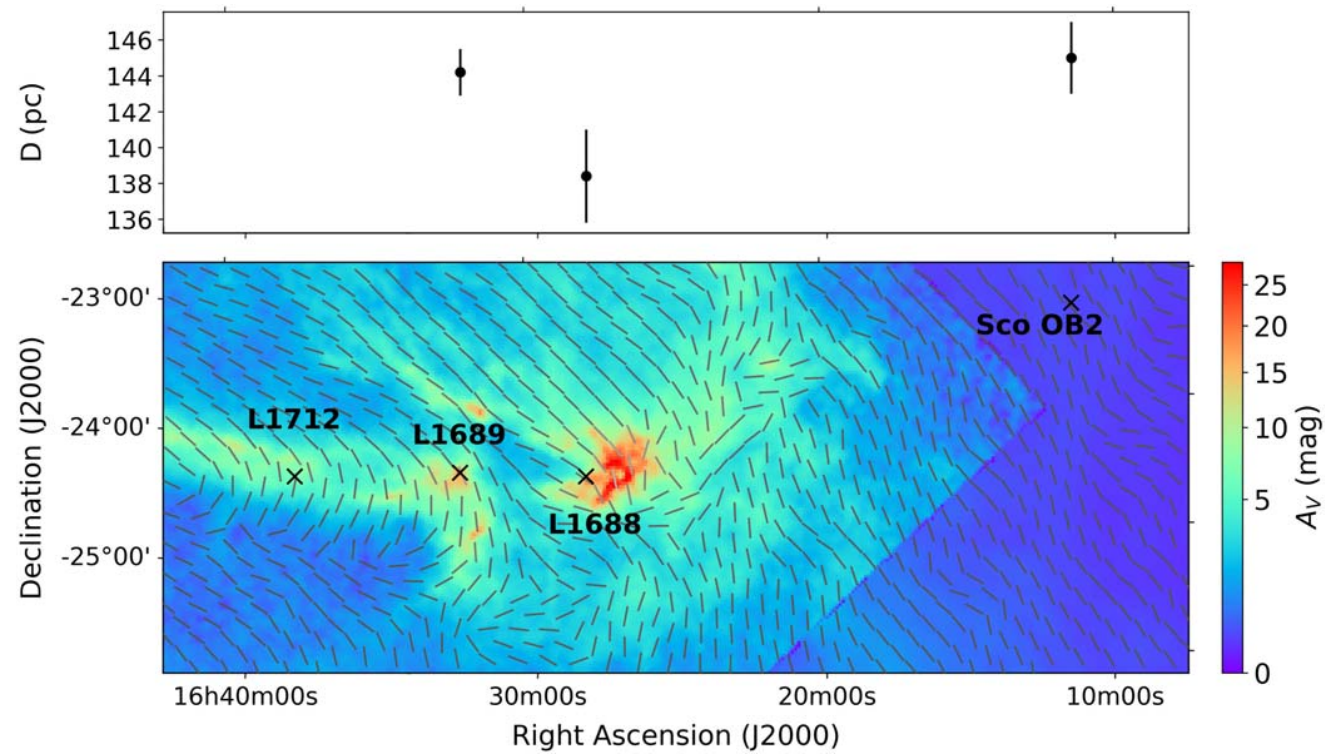

Figure 1. Main (lower) panel: the Ophiuchus molecular cloud, observed in magnitudes of visual extinction $\left(A_{V}\right)$. High-resolution $A_{V}$ mapping of L1688, L1689, and part of their filamentary streamers is taken from Two Micron All Sky Survey imaging made as part of the COMPLETE Survey (Ridge et al. 2006); extinction values in areas not covered by COMPLETE are taken from the NASA/IPAC Infrared Science Archive (Schlegel et al. 1998; Schlafly \& Finkbeiner 2011). The L1689/L1712 filamentary streamer runs approximately E-W across the northern part of L1689, turning to run approximately NE/SW, toward and beyond the L1712 region, on the eastern side of L1689 (Loren 1989). Gray half-vectors show magnetic field direction inferred from Planck $353 \mathrm{GHz}$ polarization angle measurements, with 10' spacing, drawn at a constant length and rotated by $90^{\circ}$ to trace the large-scale magnetic field direction. Crosses mark the positions used to calculate distances between the clouds and Sco OB2, as described in the text. Upper panel: line-of-sight distances to L1688, L1689, and Sco OB2 (de Zeeuw et al. 1999; Ortiz-León et al. 2018).

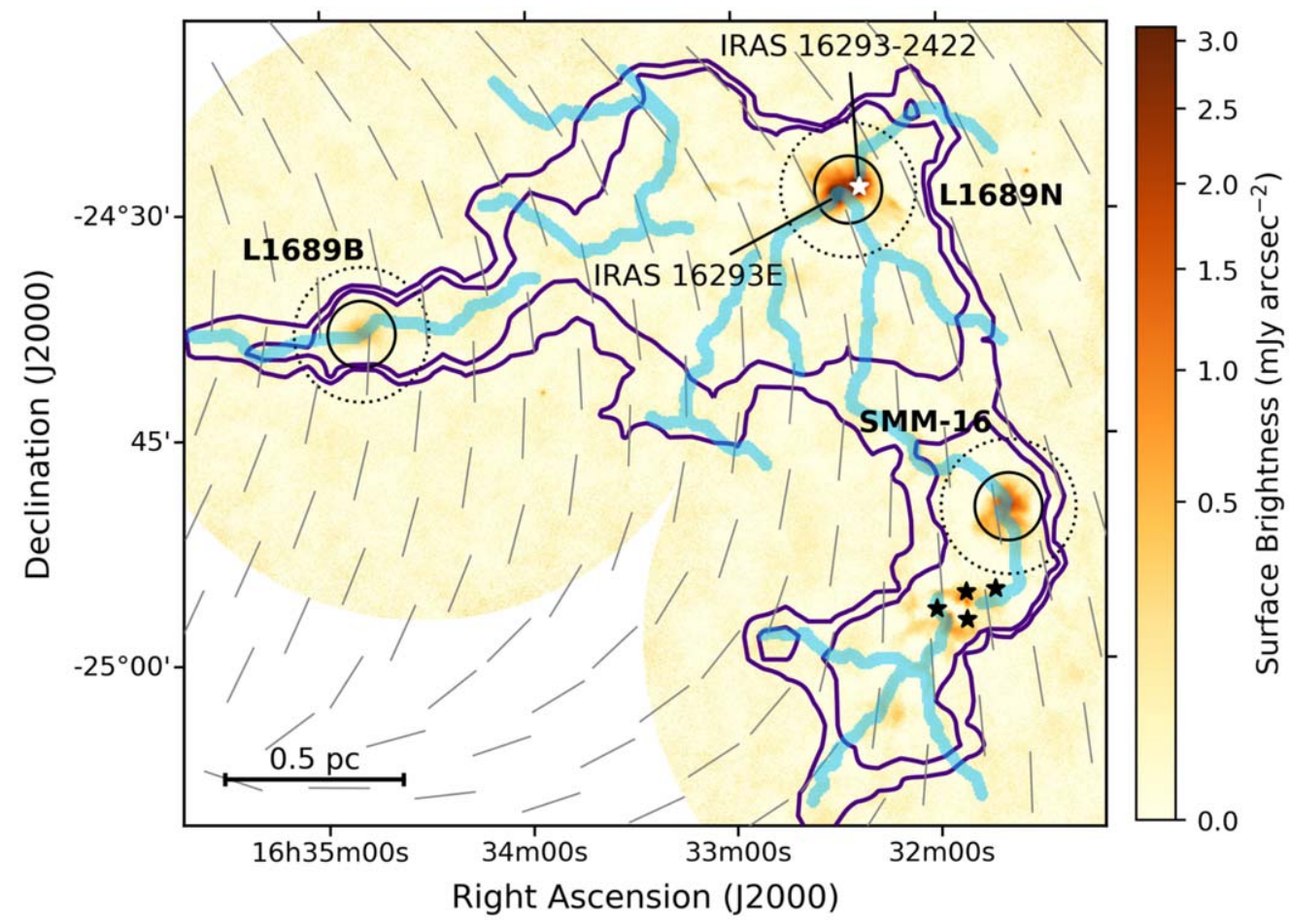

Figure 2. The L1689 molecular cloud observed in $850 \mu$ m emission with SCUBA-2 (Pattle et al. 2015) (white areas are beyond the extent of the SCUBA-2 map). Black circles mark the extent of our POL-2 observations: solid circles show the central $6^{\prime}$ region of useful coverage; dotted circles show the full extent of the observation. Gray half-vectors show Planck $353 \mathrm{GHz}$ polarization angles, drawn at a constant length and rotated by $90^{\circ}$ to trace the large-scale magnetic field direction. Purple lines mark the $A_{V}=7$ contour (Ridge et al. 2006), approximately delineating the extent of the L1689 cloud, and the $A_{V}=8$ contour enclosing both L1689N and L1689B, as part of the L1689/L1712 filamentary streamer (see Loren 1989). Blue lines mark the filamentary network identified in Herschel observations by Ladjelate et al. (2020). Stars mark embedded protostars in the region, as identified by Enoch et al. (2009). The L1689N region, the IRAS 16293-2422 protostellar core, the IRAS 16293E (SMM-19) core, the SMM-16 clump, and the L1689B core are also labeled. Physical scale is shown in the lower left corner, for an assumed distance of $144.2 \mathrm{pc}$. 
L1688 to Sco OB2 being $11.5 \pm 3.3 \mathrm{pc}$, that from L1689 to Sco OB2 being $12.2 \pm 2.4 \mathrm{pc}$, and L1688 and L1689 being located $6.3 \pm 2.9 \mathrm{pc}$ from one another. If this is the case, the differences in star formation history between L1688 and L1689 may not be attributable to their relative proximity to Sco OB2, and alternative explanations for the relatively lackluster star formation in L1689 must be sought.

The L1689 cloud, shown in Figure 2, contains two significant clumps. The northern clump, hereafter referred to as L1689N, contains the well studied protostellar system IRAS 16293-2422, a multiple system of Class 0 protostars (Wootten 1989; Mundy et al. 1992), with a quadrupolar set of outflows (Walker et al. 1988; Mizuno et al. 1990). The system contains two main sources, IRAS 16293A (south) and 16293B (north), separated by $\sim 5^{\prime \prime}$ (Chandler et al. 2005) but joined by a bridge of emission of length $\sim 700$ au (Pineda et al. 2012). The region also contains the starless core IRAS 16293E (SMM-19 in the nomenclature of Nutter et al. 2006), which is a candidate for gravitational collapse (Sadavoy et al. 2010). See Jørgensen et al. (2016) for a detailed review of the IRAS 16293 system.

The southern part of L1689, known as L1689S, contains several structures, including SMM-16 (Nutter et al. 2006), a strong candidate for being a gravitationally bound prestellar clump or core. Chitsazzadeh et al. (2014) identified SMM-16 as a starless core based on its high degree of deuterium fractionation and lack of associated infrared emission, and found it to be virially bound. However, they found no evidence for infall, instead finding SMM-16 to be oscillating. However, Pattle et al. (2015) identified three fragments, SMM-16a, b, and c, within SMM-16, suggesting that the region is a starless clump rather than a single core. Similarly, Ladjelate et al. (2020) identify three dense fragments in the center of SMM-16, and three further fragments in the periphery of the clump.

L1689 also contains the L1689B prestellar core candidate, embedded in a filamentary structure to the east of the main body of the cloud (Jessop \& Ward-Thompson 2000; Kirk et al. 2007; Steinacker et al. 2016). The core, which is generally considered to be undergoing large-scale infall (e.g., Lee et al. 2001), has been extensively studied in terms of its chemistry (e.g., Redman et al. 2002; Crapsi et al. 2005; Bacmann et al. 2016; Kim et al. 2020) and internal structure and dynamics (e.g., Lee et al. 1999, 2001; Redman et al. 2004; Seo et al. 2013; Roy et al. 2014) because of its relative isolation and simple morphology.

\section{Observations}

We observed L1689 using the POL-2 polarimeter on the SCUBA-2 camera on the JCMT. L1689N and SMM-16 were observed under project code M19AP038, and L1689B was observed as part of the JCMT BISTRO Survey (WardThompson et al. 2017) under project code M16AL004. Each field was observed 20 times in Band 2 weather $(0.05<$ $\tau_{225 \mathrm{GHz}}<0.08$ ), giving a total integration time of 14 hours per field. A single POL-2 observation consists of 40 minutes of observing time using the POL-2-DAISY scan pattern (Friberg et al. 2016), which produces a $12^{\prime}$ diameter output map, of which the central $3^{\prime}$ has approximately uniform noise, and the central $6^{\prime}$ has a useful level of coverage. The data were reduced in a two-stage process using the pol2map routine ${ }^{30}$ recently added to SMURF. ${ }^{31}$

\footnotetext{
${ }_{30}$ http://starlink.eao.hawaii.edu/docs/sun258.htx/sun258ss73.html

${ }^{31} \mathrm{http}: / /$ starlink.eao.hawaii.edu/docs/sun258.htx/sun258.html
}

In the first stage, the raw bolometer timestreams for each observation were converted into separate Stokes $Q, U$, and $I$ timestreams. An initial Stokes I map was then created from the I timestream from each observation using the iterative mapmaking routine makemap (Chapin et al. 2013). For each reduction, areas of astrophysical emission were defined using a signal-to-noise-based mask determined iteratively by makemap. Areas outside this masked region were set to zero until the final iteration of makemap (see Mairs et al. 2015 for a detailed discussion of the role of masking in SCUBA-2 data reduction). Each map was compared to the first map in the sequence to determine a set of relative pointing corrections. The individual $I$ maps were then coadded to produce an initial $I$ map of the region.

In the second stage, an improved Stokes $I$ map was created from the $I$ timestreams of each observation using makemap, and Stokes $Q$ and $U$ maps were created from their respective timestreams. The initial I map (described above) was used to generate a fixed signal-to-noise-based mask for all iterations of makemap. In the second stage, the skyloop ${ }^{32}$ routine is used, in which one iteration of makemap is performed on each of the observations in the set in turn, with the set being averaged together at the end of each iteration, rather than the observations being reduced consecutively. The pointing corrections determined in the first stage were applied to the Stokes $Q, U$, and $I$ maps during the map-making process. Correction for instrumental polarization in the Stokes $Q$ and $U$ maps was performed based on the final output $I$ map, using the "January 2018" instrumental polarization (IP) model (Friberg et al. 2018). Variances in the final coadded maps were calculated according to the standard deviation of the measured values in each pixel across the 20 observations, and in the final coadded maps, each observation was weighted according to the mean of its associated variance values. The output $Q, U$, and $I$ maps were gridded to $4^{\prime \prime}$ pixels and calibrated in mJy beam ${ }^{-1}$ using a flux conversion factor (FCF) of $725 \mathrm{Jy} \mathrm{pW}^{-1}$ (the standard SCUBA-2 $850 \mu \mathrm{m}$ FCF of $537 \mathrm{Jy} \mathrm{pW}^{-1}$ multiplied by a factor of 1.35 to account for additional losses from POL-2; Dempsey et al. 2013; Friberg et al. 2016).

Half-vector catalogs were created from the final $I, Q$, and $U$ maps, on a $12^{\prime \prime}$ pixel grid (the primary beam size of the JCMT at $850 \mu \mathrm{m}$; Dempsey et al. 2013). The term "half-vector" refers to the $\pm 180^{\circ}$ ambiguity in magnetic field direction. These catalogs list the derived polarization properties of each pixel: polarized intensity $(P)$, debiased polarized intensity $\left(P_{\mathrm{db}}\right)$, polarization fraction $(p)$, debiased polarization fraction $\left(p_{\mathrm{db}}\right)$, and polarization angle $\left(\theta_{P}\right)$, and uncertainties on each quantity. The formulae for these derived quantities are given in Appendix A. The average rms noise in Stokes $Q$ and $U$ on $12^{\prime \prime}$ pixels over the central $3^{\prime}$ of the map is $0.83 \mathrm{mJy}^{-1}$ beam $^{-1}$ in L1689N, 0.81 mJy beam $^{-1}$ in SMM-16, and 0.75 mJy beam $^{-1}$ in L1689B. The maps and half-vector catalogs used in this work are available at https://doi.org/10.11570/20.0013.

In the case of optically thin polarized emission from dust grains aligned with their minor axes parallel to the magnetic field direction, polarization angles must be rotated by $90^{\circ}$ in order to trace the plane-of-sky magnetic field direction. In the following discussion we denote magnetic field angle as $\theta$. POL2 polarization half-vector maps for L1689N, SMM-16, and L1689B are shown in Figure 3, while magnetic field half-vector

\footnotetext{
${ }^{32}$ http://starlink.eao.hawaii.edu/docs/sun258.htx/sun258ss72.html
} 


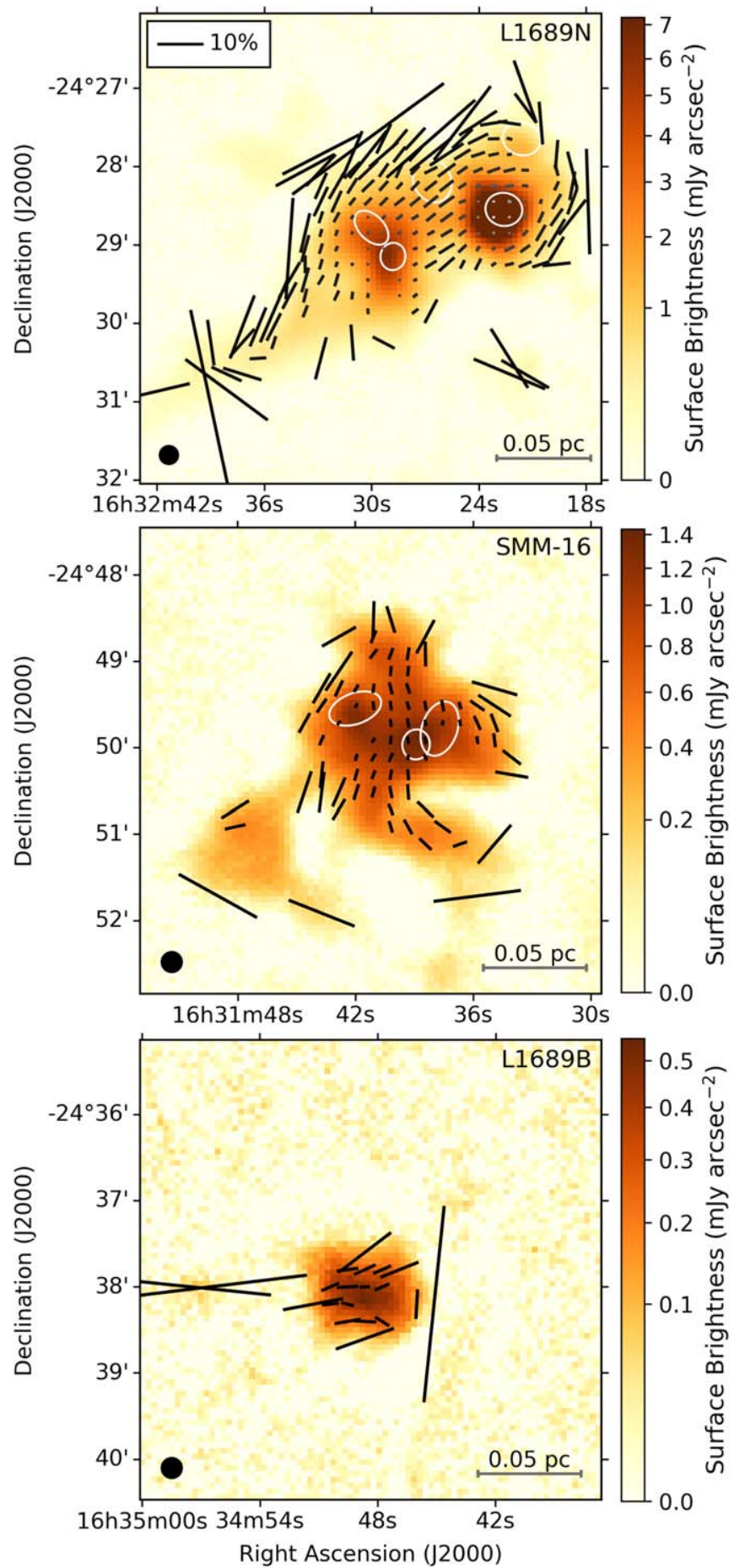

Figure 3. Debiased polarization half-vectors in L1689N (top), SMM-16 (middle), and L1689B (bottom), overlaid on POL-2 Stokes I data. POL-2 data are shown as black half-vectors (except in L1689N, where half-vectors are colored by total intensity for contrast), with selection criteria $p_{\mathrm{db}} / \delta p>3$, $I / \delta I>5$, and $\delta \theta<10^{\circ}$. Sources identified by Pattle et al. (2015) are marked as white circles: in L1689N, their sources SMM-19 and -22 overlap in the IRAS 16293E core, their SMM-20 corresponds to IRAS 16293-2422, and their SMM-23 and -24 are in the clump periphery. Their sources SMM-16a, -16b, and -16 are shown in SMM-16. The JCMT beam size is shown in the lower left corner, and a physical scale bar is shown in the lower right corner, of each plot.

maps are shown in Figure 4. Histograms of magnetic field angle are shown in Figure 5 (position angles are given in the range $-90 \leqslant \theta<+180^{\circ}$, where the ranges $-90^{\circ}-0^{\circ}$ and

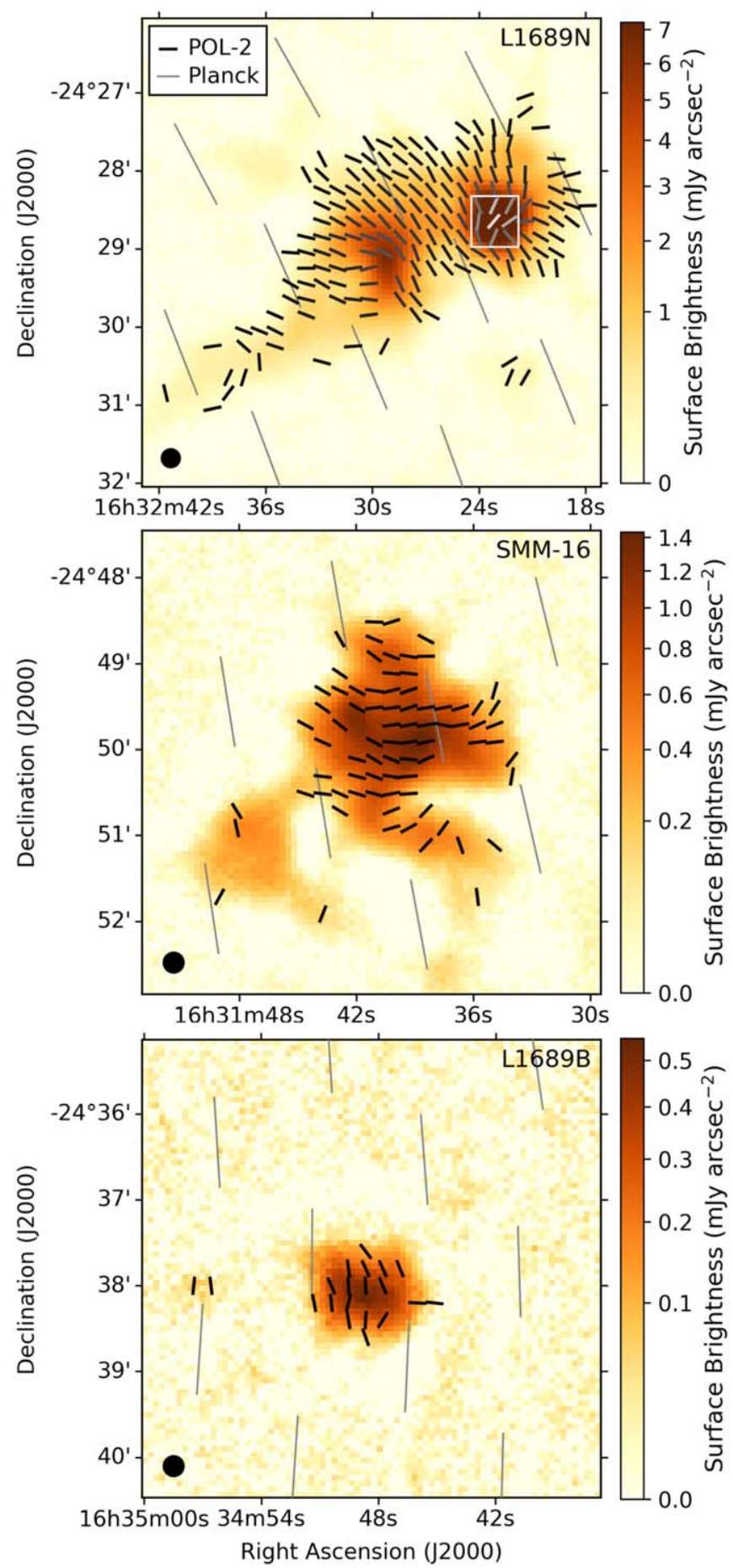

Figure 4. Magnetic field half-vectors (polarization half-vectors rotated by $90^{\circ}$ ) in L1689N (top), SMM-16 (middle), and L1689B (bottom), overlaid on POL-2 Stokes $I$ data. POL-2 data are shown as black half-vectors of uniform length (except in L1689N, where half-vectors are colored by total intensity for contrast) with selection criteria as in Figure 3. Planck data are shown as gray half-vectors. The JCMT beam size is shown in the lower left corner of each plot. (Note that the Planck half-vectors shown in this image are oversampled.) The half-vectors in L1689N associated with IRAS 16293-2422 are enclosed in a white box.

$+90^{\circ}-+180^{\circ}$ are identical). In these figures and in all subsequent analysis (except where noted in Section 5), we select half-vectors where $I / \delta I>5, p_{\mathrm{db}} / \delta p>3$, and $\delta \theta<10^{\circ}$, 

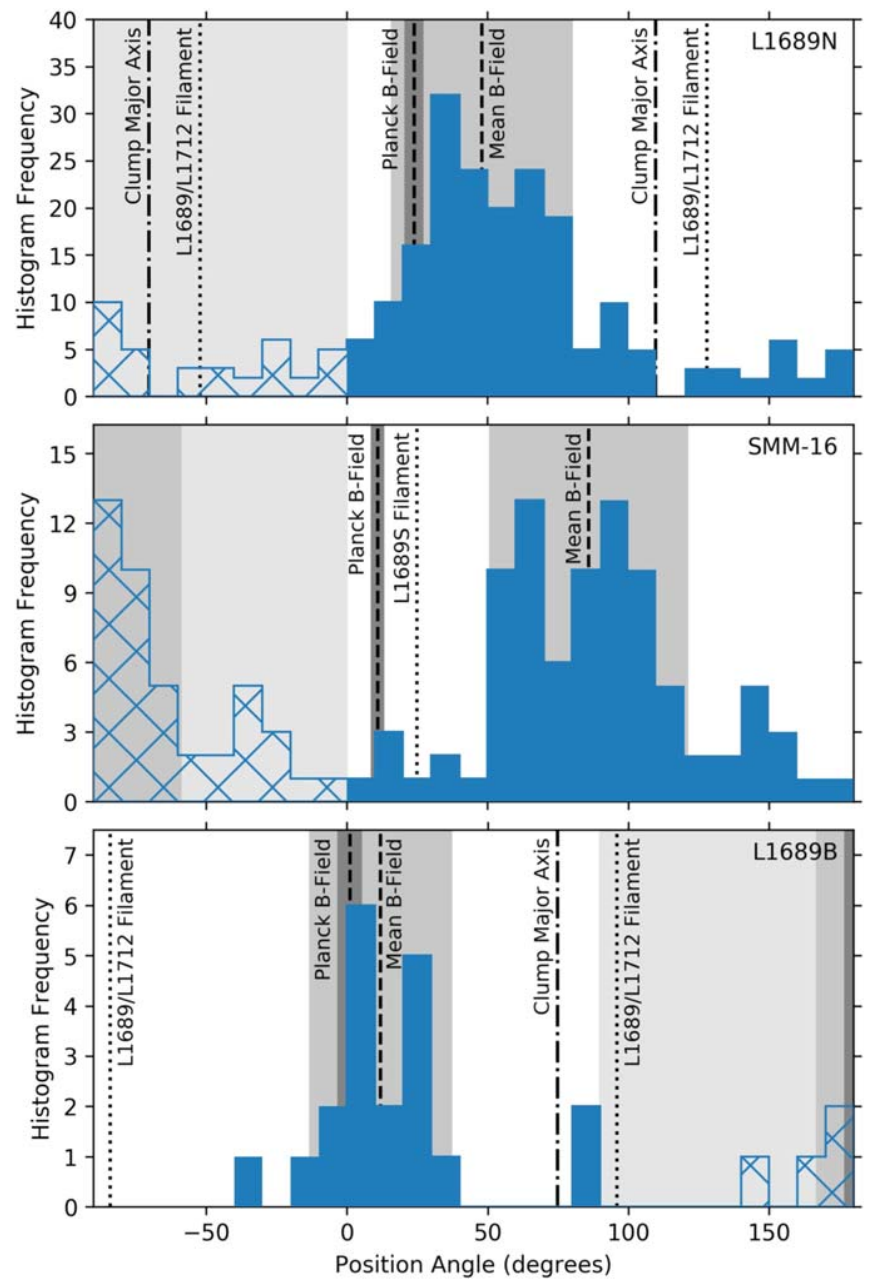

Figure 5. Distribution of magnetic field position angles in L1689N (top), SMM-16 (middle), and L1689B (bottom). Position angles are given in degrees $\mathrm{E}$ of $\mathrm{N}$, in the range $-90 \leqslant \theta<+180^{\circ}$. The ranges $-90^{\circ}-0^{\circ}$ and $+90^{\circ}-$ $+180^{\circ}$ are identical. For L1689N and SMM-16, we emphasize the range $0-+180^{\circ}$, while for L1689B we emphasize the range $-90-+90^{\circ}$, in each case chosen to best illustrate the typical magnetic field direction and the distribution of the magnetic field angles. The duplicated range is shaded in light gray in each panel, with position angles plotted in hatched histogram bins. In each panel, black dashed lines mark the mean POL-2 and Planck position angles; shaded gray areas show their standard deviations. Black dotted-dashed lines mark the fitted major-axis position angle of the clump/core as listed in Table 1, with the exception of SMM-16, which does not have a well defined major axis. Dotted lines mark the approximate major-axis position angle of the filament in which the clump/core is embedded, as determined from Herschel measurements (Ladjelate et al. 2020).

noting that Serkowski's approximation $(\delta \theta \approx 28.65 \times(\delta p / p)$; Serkowski 1962) makes the latter two criteria approximately equivalent to one another.

\section{Magnetic Field Properties in L1689}

\subsection{Magnetic Field Morphology}

\subsection{1. $L 1689 N$}

L1689N has a well ordered magnetic field that is aligned broadly $\mathrm{NE} / \mathrm{SW}$, with a mean direction $48^{\circ} \pm 32^{\circ} \mathrm{E}$ of $\mathrm{N}$ (calculated over the circular range $0^{\circ}-180^{\circ}$ ). The exception to this is at the position of the IRAS 16293-2422 protostar itself, where the inferred magnetic field direction is $166^{\circ} \pm 31^{\circ} \mathrm{E}$ of $\mathrm{N}$, measured over the nine independent pixels covering a $36^{\prime \prime} \times 36^{\prime \prime}$ area centered on the protostar. Excluding IRAS $16293-2422$, the mean field direction is $50^{\circ} \pm 90^{\circ} \mathrm{E}$ of $\mathrm{N}$, so that the polarization half-vectors of IRAS 16293-2422 are $64^{\circ} \pm 43^{\circ}$ offset from the mean elsewhere in L1689N. The half-vectors associated with IRAS 16293-2422 are marked on Figure 4.

Away from IRAS 16293-2422, the magnetic field inferred from POL-2 observations is ordered and generally linear, with a mean direction $50^{\circ} \pm 30^{\circ} \mathrm{E}$ of $\mathrm{N}$. We see some deviations from the mean field direction in the SE and NW edges of the clump, particularly in the vicinity of IRAS 16293E.

The mean field direction in $\mathrm{L} 1689 \mathrm{~N}$ is similar to the field observed on arcminute scales by Planck $\left(\left\langle\theta_{\text {Planck }}\right\rangle=24^{\circ} \pm 3^{\circ}\right)$, and approximately perpendicular to $\left(\sim 78^{\circ}\right.$ offset from) the major axis of the L1689/L1712 filament of which the clump is a part, which runs $\sim 128^{\circ} \mathrm{E}$ of $\mathrm{N}$ (see Ladjelate et al. 2020, see also Figures 2 and 5).

Although the L1689N clump is at the junction of two filaments, one running approximately $\mathrm{SE} / \mathrm{NW}$ and the other approximately $\mathrm{NE} / \mathrm{SW}$, as shown in Figures 2 and 6, we consider L1689N to be principally associated with the SE/NW filament, which extends over several degrees as part of the L1689/L1712 filamentary streamer, as shown in Figure 1. L1689N and L1689E are embedded in the same larger structure, with both enclosed in the same $A_{V}=8$ contour, as shown in Figure 2, and this structure extends west beyond the extent of L1689N that we observe. This filament is also detectable in our Stokes $I$ observations, unlike the NE/SW filament with lower column density, as shown in Figure 6. Moreover, the major axis of L1689N is well aligned with the major axis of the SE/NW filament, as shown in Figures 5 and 6 (clump orientation is determined as described in Section 4.2, below). However, an alternative interpretation is that L1689N is located at the point where the main L1689/L1712 filamentary streamer turns to become the short extension to L1689S. In this case, the major axis of the filament at the position of L1689N could be defined as the average of the SE/ $\mathrm{NW}$ and NE/SW filament directions. The NE/SW filament is oriented $\sim 27^{\circ} \mathrm{E}$ of $\mathrm{N}$, and so the average orientation of the filaments at the junction of which L1689N is embedded in this interpretation is $\sim 78^{\circ} \mathrm{E}$ of $\mathrm{N}$. This is again significantly offset from the plane-of-sky magnetic field direction that we infer.

At the position of IRAS 16293-2422, the magnetic field inferred from POL-2 observations flips by $\sim 64^{\circ}$ relative to the large-scale field, appearing to run approximately $\mathrm{NW} / \mathrm{SE}$. Polarization observations of protostellar disks from the Atacama Large Millimeter/submillimeter Array (ALMA) have shown abrupt changes in polarization angle (e.g., Ko et al. 2020). Mechanisms proposed to explain this change include scattering from large dust grains (Kataoka et al. 2015; Sadavoy et al. 2019), intrinsic polarization due to thermal emission from nonspherical dust grains (Kirchschlager et al. 2019; Guillet et al. 2020), and the effects of optically thick emission (Ko et al. 2020). However, in IRAS 16293-2422 the JCMT beam encompasses not only two differently-inclined protostellar disks but also the protobinary envelope and its internal dust structures (Pineda et al. 2012). The magnetic field direction seen in our observations corresponds well with the magnetic field direction seen by ALMA in the bridge of dense gas that 


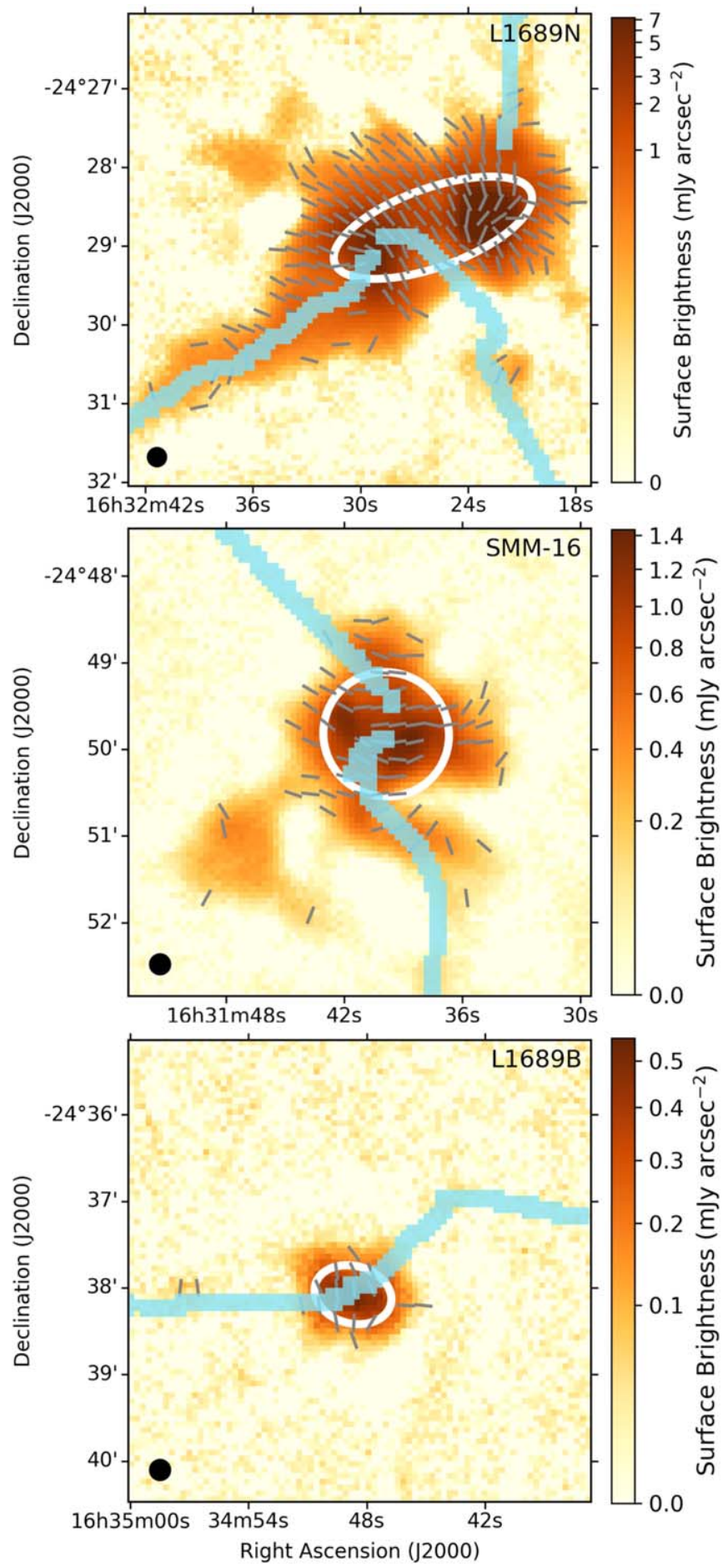

Figure 6. POL-2 magnetic field half-vectors in L1689N (top), SMM-16 (middle), and L1689B (bottom), overlaid on POL-2 Stokes $I$ data, as in Figure 3. The FWHM best-fit ellipses to the Stokes $I$ emission are shown as white ellipses. The filaments identified in Herschel observations by Ladjelate et al. (2020) are shown as blue lines. The L1689N Stokes $I$ data are plotted using a logarithmic color scale, to emphasize the faint larger-scale structure.

connects the IRAS 16293A and B disks (Sadavoy et al. 2018), as discussed in Section 6.1 below. This good agreement with ALMA observations suggests that our observations of IRAS 16293-2422 trace dust grains that are both optically thin and aligned with respect to the magnetic field, and so we consider our observations to be tracing the magnetic field throughout L1689N.

\subsection{2. $S M M-16$}

SMM-16 has a fairly well ordered magnetic field, which is aligned approximately $\mathrm{E} / \mathrm{W}$ in the densest parts of the clump, but which shows significant deviations in its outer regions (mean direction: $86^{\circ} \pm 35^{\circ}$ ). The inferred field direction tends toward a NE/SW direction in the eastern side of the clump, and to a SE/NW direction in the western side. The average magnetic field direction is approximately perpendicular both to the Planck-scale magnetic field direction, which runs $\mathrm{N}-\mathrm{S}$ across the region $\left(\left\langle\theta_{\text {Planck }}\right\rangle=11^{\circ} \pm 2^{\circ}\right)$, and to the orientation of the filament in which the clump is embedded, which runs $\sim 25^{\circ} \mathrm{E}$ of $\mathrm{N}$ (see Ladjelate et al. 2020, see also Figures 2 and 5). Similar discrepancies between POL-2 and Planck-scale fields have recently been seen in BISTRO survey observations of Perseus NGC 1333 (Doi et al. 2020). The distribution of POL-2 magnetic field half-vectors that we observe is thus suggestive of a field that is perpendicular to the large-scale field in the dense center of the clump, with significant excursions in the lower-density periphery. This perpendicular component is represented by the main peak in Figure 5, and is more clearly apparent in Figures 9 and 10 in Appendix B. We further tentatively suggest that in the lower-density periphery of the eastern and western sides of the clump, the field may curve to join the large-scale field in the lower-density surrounding region, as is suggested by the peaks at $65^{\circ}$ and $150^{\circ} \mathrm{E}$ of $\mathrm{N}$ in Figure 5, representing ordered field structure on the eastern and western sides of the core respectively.

\subsection{3. $L 1689 B$}

L1689B is considerably smaller and fainter than the other two clumps, and consequently fewer reliable polarization halfvectors are detected. Nonetheless, we observe a consistent magnetic field running broadly $\mathrm{N} / \mathrm{S}$ across the core, with the exception of two half-vectors on the western edge of the core. The mean field direction is $12^{\circ} \pm 25^{\circ}$. The Planck-scale field in the region also runs $\mathrm{N} / \mathrm{S}\left(\left\langle\theta_{\text {Planck }}\right\rangle=1^{\circ} \pm 4^{\circ}\right)$, similar to the mean field direction that we observe. The field that we observe with POL-2 is again approximately perpendicular to the filament in which the core is embedded, which runs $\sim 96^{\circ} \mathrm{E}$ of N, approximately E/W (see Kirk et al. 2007; Ladjelate et al. 2020, see also Figures 2 and 5).

\subsubsection{Comparison between Regions}

The discrepant plane-of-sky POL-2 and Planck-scale fields in SMM-16 contrast notably with L1689N and L1689B, in which the POL-2- and Planck-scale fields agree well in projection on the plane of the sky. However, in all three clumps the POL-2-scale magnetic field is perpendicular to the local filament direction.

\subsection{Davis-Chandrasekhar-Fermi Analysis}

We estimated magnetic field strengths in L1689 using the Davis-Chandrasekhar-Fermi (DCF) method (Davis 1951; Chandrasekhar \& Fermi 1953), which assumes that perturbations in the magnetic field are Alfvénic; i.e., deviation in angle from the mean field direction is due to distortion by small-scale nonthermal motions, and the Alfvén Mach number of the gas is given by

$$
\mathcal{M}_{A}=\frac{\sigma_{\mathrm{NT}}}{v_{A}}=\frac{\sigma_{\theta}}{Q},
$$


where $\sigma_{\theta}$ is the dispersion in magnetic field angle in radians, $Q$ is a correction factor for line-of-sight and sub-beam integration effects, such that $0<Q<1$ (Ostriker et al. 2001), $\sigma_{\mathrm{NT}}$ is the nonthermal velocity dispersion of the gas, and $v_{A}$ is the Alfvén velocity of the magnetic field, which is then given in cgs units by

$$
v_{A}=\frac{B}{\sqrt{4 \pi \rho}}=Q \frac{\sigma_{\mathrm{NT}}}{\sigma_{\theta}},
$$

where $B$ is magnetic field strength and $\rho$ is gas density. This equation can then be rearranged to give an expression for plane-of-sky magnetic field strength $B_{\text {pos}}$, noting that the value of $\sigma_{\theta}$ that we measure represents only the plane-of-sky component of the angle dispersion,

$$
B_{\mathrm{pos}} \approx Q \sqrt{4 \pi \rho} \frac{\sigma_{\mathrm{NT}}}{\sigma_{\theta}} .
$$

Crutcher et al. (2004) note that on average $B_{\mathrm{pos}} / B \approx \pi / 4$. Using plane-of-sky angular dispersion will also cause $v_{A}$ to be correspondingly underestimated, and $\mathcal{M}_{A}$ to be overestimated. However, as the correction factor of Crutcher et al. (2004) can be meaningfully applied only to a statistical ensemble of measurements, we do not apply it in this work, noting that its inclusion would not alter any of our conclusions.

We use the formulation of Equation (3) given by Crutcher et al. (2004),

$$
B_{\mathrm{pos}}(\mu \mathrm{G}) \approx 9.3 \sqrt{n\left(\mathrm{H}_{2}\right)\left(\mathrm{cm}^{-3}\right)} \frac{\Delta v_{\mathrm{NT}}\left(\mathrm{km} \mathrm{s}^{-1}\right)}{\sigma_{\theta}(\mathrm{deg})},
$$

where number density $n\left(\mathrm{H}_{2}\right)=\rho / \mu m_{\mathrm{H}}$ and FWHM nonthermal velocity dispersion $\Delta v_{\mathrm{NT}}=\sigma_{\mathrm{NT}} \sqrt{8 \ln 2}$. Note that this formulation takes $Q=0.5$ (see Heitsch et al. 2001; Ostriker et al. 2001) and assumes a mean molecular weight $\mu=2.8$, which we adopt throughout this work. Equation (4) is equivalent to the following relations: for Alfvén Mach number,

$$
\mathcal{M}_{A} \approx 3.5 \times 10^{-2} \sigma_{\theta}(\mathrm{deg}),
$$

and for Alfvén velocity,

$$
v_{A}\left(\mathrm{~km} \mathrm{~s}^{-1}\right) \approx 12.1 \frac{\Delta v_{\mathrm{NT}}\left(\mathrm{km} \mathrm{s}^{-1}\right)}{\sigma_{\theta}(\operatorname{deg})} .
$$

We outline here our approach to DCF analysis in L1689. A detailed description of how the values that we use are derived is given in Appendix B.

We estimated the size of each of the clumps by fitting a 2D Gaussian distribution to the emission associated with the clump. In the case of L1689N, we masked emission in the 40" diameter region surrounding the IRAS 16293-2422 protostar, because we aim to investigate the magnetic field strength in the larger clump, rather than the behavior of IRAS 16293-2422 itself, the magnetic field of which appears to be behaving quite differently to that in the clump in which it is embedded (see Section 6.1). Polarization half-vectors in this region are excluded from the determination of angle dispersions, and flux from this region is included in mass and density calculations only where noted. The best-fit Gaussian distributions are listed in Table 1 and marked on Figure 6.

We measured flux densities $F_{\nu}$ via aperture photometry over an elliptical aperture of diameter 1 FWHM for each region. We measured dispersion in polarization angle $\sigma_{\theta}$ over the same
Table 1

Gaussian Fits to Stokes I Emission from L1689N, SMM-16, and L1689B

\begin{tabular}{lccc}
\hline \hline & L1689N & SMM-16 & L1689B \\
\hline Peak value (mJy arcsec & & \\
Center R.A. (hh:mm:ss.ss) & 3.00 & 0.95 & 0.53 \\
Center decl. (dd:mm:ss.s) & $-24: 28: 46.2$ & $-24: 49: 49.7$ & $-24: 38: 04.7$ \\
$a$ (major std. dev.) (arcsec) & 67.4 & 37.1 & 22.9 \\
$b$ (minor std. dev.) (arcsec) & 25.5 & 36.4 & 16.9 \\
P.A. (deg E of N) & 109.8 & 56.3 & 74.9 \\
$R$ (pc) & 0.056 & 0.030 & 0.019 \\
\hline
\end{tabular}

Note. Note that IRAS 16293-2422 was masked as shown in Figure 9.

areas, by fitting a Gaussian model in L1689N and SMM-16, and through direct calculation in L1689B. The values for each region are listed in Table 2.

We further defined a representative half-width at halfmaximum radius for each source,

$$
R=D \sqrt{2 \ln 2 \tan a \tan b},
$$

where $a$ is the major-axis Gaussian width, $b$ is the minor-axis Gaussian width, and $D=144.2 \mathrm{pc}$ is the distance to L1689. This radius, also listed in Table 1 , is used in calculations of column and volume density and in Section 4.3 below.

Masses for each source were calculated using the relation of Hildebrand (1983),

$$
M=\frac{F_{\nu} D^{2}}{\kappa_{\nu} B_{\nu}(T)},
$$

where dust opacity $\kappa_{\nu}=0.0125 \mathrm{~cm}^{2} \mathrm{~g}^{-1}$ and $B_{\nu}(T)$ is the Planck function. We take temperature $T=12 \mathrm{~K}$ for all of the sources, and conservatively assume a systematic uncertainty of $50 \%$ on $\kappa_{\nu}$ (see Roy et al. 2014). Column density is calculated as

$$
N\left(\mathrm{H}_{2}\right)=\frac{M}{\mu m_{\mathrm{H}}} \frac{1}{\pi R^{2}},
$$

and volume density as

$$
n\left(\mathrm{H}_{2}\right)=\frac{M}{\mu m_{\mathrm{H}}} \frac{3}{4 \pi R^{3}} .
$$

Gas FWHM velocity dispersion values $\Delta v$ were taken from $\mathrm{N}_{2} \mathrm{H}^{+} J=1 \rightarrow 0$ measurements made by Pan et al. (2017) in the case of L1689N and SMM-16, and by Lee et al. (2001) in the case of L1689B, and corrected for the thermal linewidth component to give $\Delta v_{\mathrm{NT}}$. The flux densities $\left(F_{\nu}\right)$, masses $(M)$, column and volume densities $\left(N\left(\mathrm{H}_{2}\right)\right.$ and $n\left(\mathrm{H}_{2}\right)$ respectively), FWHM velocity dispersions $\left(\Delta v_{\mathrm{NT}}\right)$, mean magnetic field angle $(\langle\theta\rangle)$, and magnetic field angle dispersion $\left(\sigma_{\theta}\right)$ determined for each clump are listed in Table 2.

\subsubsection{Alfvén Mach Number}

Using Equation (5), we find that $\mathcal{M}_{A}=0.23 \pm 0.05$ in L1689N, while $\mathcal{M}_{A}=0.34 \pm 0.06$ in SMM-16 and $\mathcal{M}_{A}=$ $0.47 \pm 0.26$ in L1689B. All three clumps have sub-Alfvénic nonthermal motions, suggesting that magnetic fields are more important to clump stability than are nonthermal motions. This result follows from the ordered field morphologies seen in the clumps. 
Table 2

Quantities Relevant to Calculations of Magnetic Field Strength

\begin{tabular}{|c|c|c|c|c|}
\hline & \multicolumn{2}{|c|}{ L1689N } & \multirow{2}{*}{ SMM-16 } & \multirow{2}{*}{ L1689B } \\
\hline & All & With Masking & & \\
\hline$F_{\nu}(\mathrm{Jy})$ & 45.14 & 23.62 & 5.41 & 0.64 \\
\hline$M\left(M_{\odot}\right)$ & $17.2 \pm 14.4$ & $9.0 \pm 7.5$ & $2.1 \pm 1.4$ & $0.24 \pm 0.17$ \\
\hline$N\left(\mathrm{H}_{2}\right)\left(\mathrm{cm}^{-2}\right)$ & $(7.9 \pm 6.6) \times 10^{22}$ & $(4.2 \pm 3.5) \times 10^{22}$ & $(3.3 \pm 2.2) \times 10^{22}$ & $(9.8 \pm 7.7) \times 10^{21}$ \\
\hline$n\left(\mathrm{H}_{2}\right)\left(\mathrm{cm}^{-3}\right)$ & $(3.5 \pm 2.9) \times 10^{5}$ & $(1.8 \pm 1.5) \times 10^{5}$ & $(2.7 \pm 1.8) \times 10^{5}$ & $(1.3 \pm 1.0) \times 10^{5}$ \\
\hline$\Delta v_{\mathrm{NT}}\left(\mathrm{km} \mathrm{s}^{-1}\right)$ & \multicolumn{2}{|c|}{$0.61 \pm 0.04$} & $0.57 \pm 0.04$ & $0.29 \pm 0.03$ \\
\hline$\langle\theta\rangle(\operatorname{deg})$ & $\cdots$ & $34.2 \pm 0.8$ & $95.9 \pm 0.4$ & 8.4 \\
\hline$\sigma_{\theta}(\mathrm{deg})$ & $\cdots$ & $6.6 \pm 0.6$ & $9.7 \pm 0.4$ & $13.3 \pm 4.7$ \\
\hline $\mathcal{M}_{A}$ & $\cdots$ & $0.23 \pm 0.05$ & $0.34 \pm 0.06$ & $0.47 \pm 0.26$ \\
\hline$v_{A}\left(\mathrm{~km} \mathrm{~s}^{-1}\right)$ & $\ldots$ & $1.12 \pm 0.17$ & $0.72 \pm 0.08$ & $0.26 \pm 0.12$ \\
\hline$B_{\text {pos }}\left(\right.$ fiducial $\left.\kappa_{\nu}\right)(\mu \mathrm{G})$ & $\cdots$ & $366 \pm 55$ & $284 \pm 31$ & $72 \pm 33$ \\
\hline$B_{\text {pos }}$ (full range) $(\mu \mathrm{G})$ & $\cdots$ & $157-575$ & $160-408$ & $12-132$ \\
\hline$\lambda\left(\right.$ fiducial $\left.\kappa_{\nu}\right)$ & $1.52 \pm 0.23$ & $0.79 \pm 0.12$ & $0.81 \pm 0.09$ & $0.95 \pm 0.44$ \\
\hline$\lambda$ (full range) & $0.66-2.38$ & $0.34-1.24$ & $0.47-1.15$ & $0.14-1.76$ \\
\hline
\end{tabular}

Note. Quantities shown: flux density $\left(F_{\nu}\right)$, mass $(M)$, column density $\left(N\left(\mathrm{H}_{2}\right)\right)$, volume density $\left(n\left(\mathrm{H}_{2}\right)\right)$, FWHM nonthermal velocity dispersion $\left(v_{\mathrm{FWHM}}\right)$, mean magnetic field angle $(\langle\theta\rangle)$, dispersion in magnetic field angle $\left(\sigma_{\theta}\right)$, Alfvén Mach number $\left(\mathcal{M}_{A}\right)$, Alfvén velocity $\left(v_{A}\right)$, plane-of-sky magnetic field strength $\left(B_{\text {pos }}\right)$, and mass-to-flux ratio $(\lambda)$, in the latter two cases listing both the fiducial- $\kappa_{\nu}$ value and its statistical uncertainty, and the full range of results including systematic uncertainty on density. When calculating masked values in L1689N, $F_{\nu}, M, N\left(\mathrm{H}_{2}\right)$, and $n\left(\mathrm{H}_{2}\right)$ were calculated assuming a constant flux density over the masked area covering IRAS 16293-2422 as described in Appendix B, and all magnetic field half-vectors associated with the masked area were excluded from determination of $\langle\theta\rangle$ and $\sigma_{\theta} . \Delta v_{\mathrm{NT}}$ values are taken from Pan et al. (2017) for L1689N and SMM-16, and from Lee et al. (2001) for L1689B, and are corrected for the thermal component of the linewidth. Mean position angle and dispersion in position angle are taken from Gaussian fitting for L1689N and SMM-16, and calculated directly for L1689B.

\subsubsection{Alfvén Velocity}

Using Equation (6), we find that $v_{A}=1.12 \pm 0.17 \mathrm{~km} \mathrm{~s}^{-1}$ in L1689N, while $v_{A}=0.72 \pm 0.06 \mathrm{~km} \mathrm{~s}^{-1}$ in SMM-16 and $v_{A}=$ $0.26 \pm 0.12 \mathrm{~km} \mathrm{~s}^{-1}$ in L1689B.

\subsubsection{Magnetic Field Strength}

Combining these measurements using Equation (4), we estimated plane-of-sky magnetic field strengths of $366 \pm$ $209 \mu \mathrm{G}$ in L1689N, $284 \pm 127 \mu \mathrm{G}$ in SMM-16, and $72 \pm$ $61 \mu \mathrm{G}$ in L1689B. We emphasize that the uncertainties on these values are predominantly systematic, being dominated by systematic uncertainty on $n\left(\mathrm{H}_{2}\right)$, itself predominantly caused by uncertainty on $\kappa_{\nu}$ (see Equations (8) and (10)). These measurements are more meaningfully described as being in the range $\sim 160-580 \mu \mathrm{G}$ in $\mathrm{L} 1689 \mathrm{~N}, \sim 160-410 \mu \mathrm{G}$ in SMM-16, and $\sim 10-130 \mu \mathrm{G}$ in L1689B. The magnetic field strengths at our fiducial value of $\kappa_{\nu}$ are $366 \pm 55 \mu \mathrm{G}, 284 \pm 31 \mu \mathrm{G}$, and $72 \pm 33 \mu \mathrm{G}$ in L1689N, SMM-16, and L1689B respectively.

The magnetic field strength values in L1689N and SMM-16 are quite large, indicating a significant enhancement of the magnetic field strength over that of the diffuse ISM (median strength $6.0 \pm 1.8 \mu \mathrm{G}$; Heiles \& Troland 2005), but comparable to some previous measurements of magnetic field strength in nearby low-mass star-forming and starless clumps and cores inferred from JCMT observations. Crutcher et al. (2004) measured plane-of-sky magnetic field strengths of $140 \mu \mathrm{G}$, $80 \mu \mathrm{G}$. and $160 \mu \mathrm{G}$ in the starless cores L1544, L183, and L43, respectively. These values are also comparable to recent measurements in L1688 made using POL-2. Kwon et al. (2018) measured field strengths in the range $200-5000 \mu \mathrm{G}$ in the star-forming clump Oph A, while Soam et al. (2018) measured 220-1040 $\mu \mathrm{G}$ in the star-forming clump Oph B, and Liu et al. (2019) measured 103-213 $\mu \mathrm{G}$ in the starless clump/ core Oph $\mathrm{C}$. The highly ordered magnetic field morphologies seen in Figure 4 are also consistent with a relatively strong magnetic field. The magnetic field strength of L1689B is somewhat weaker, comparable to the magnetic field strengths measured in starless cores by Crutcher et al. (2004), but also to those measured in the isolated starless cores L1498 and L1517B, which were found by Kirk et al. (2006) to have magnetic field strengths of $\sim 10 \mu \mathrm{G}$ and $\sim 30 \mu \mathrm{G}$ respectively.

\subsubsection{Mass-to-flux Ratio}

The relative importance of magnetic fields and gravity in a clump or core can be characterized using the mass-to-flux ratio $\lambda$, the ratio of measured mass to the maximum mass that could be supported against collapse under self-gravity by the measured magnetic flux. A magnetically subcritical object, $\lambda<1$, is magnetically supported, while a magnetically supercritical object, $\lambda>1$, is gravitationally unstable.

We estimated the mass-to-flux ratio $\lambda$ using the formulation of Crutcher et al. (2004),

$$
\lambda=7.6 \times 10^{-21} \frac{N\left(\mathrm{H}_{2}\right)\left(\mathrm{cm}^{-2}\right)}{B_{\mathrm{pos}}(\mu \mathrm{G})} .
$$

In $\mathrm{L} 1689 \mathrm{~N}$ we measured $\lambda=0.79 \pm 0.45$ (for fiducial $\kappa_{\nu}$, $\lambda=0.79 \pm 0.12)$ when IRAS 16293-2422 is masked, and $\lambda=1.52 \pm 0.86$ (fiducial $\kappa_{\nu}: \lambda=1.52 \pm 0.23$ ) using the total mass, while in SMM-16 we measured $\lambda=0.81 \pm 0.34$ (fiducial $\kappa_{\nu}: \lambda=0.81 \pm 0.09$ ), and in L1689B $\lambda=0.95 \pm 0.81$ (fiducial $\left.\kappa_{\nu}: \lambda=0.95 \pm 0.44\right)$. These values suggest that all of the clumps are magnetically transcritical-i.e., either they are marginally supported against collapse by their internal magnetic fields or they are marginally gravitationally unstable, but magnetic fields are dynamically important in the clumps' evolution. However, we note that the validity of a comparison of plane-of-sky magnetic field strength to line-of-sight column 
density is uncertain; our values of $\lambda$ could be overestimated by a factor of $\sim 1.33-3$ depending on the three-dimensional geometry of the clumps (Crutcher et al. 2004; Planck Collaboration et al. 2016). We discuss the interpretation of these values further in Section 6, but note that the values of $\lambda$ determined here are best considered as a qualitative indicator that these clumps are approximately magnetically critical rather than as a precise measure of their stability against collapse.

\subsection{Energy Balance}

We calculated the energy balance for each of our clumps using the properties derived above.

\subsubsection{Gravitational Potential Energy}

The gravitational potential energy (GPE) of a spherically symmetric density distribution is given by

$$
E_{G}=-\eta \frac{G M^{2}}{R} .
$$

The value of the constant $\eta$, and so of $E_{G}$, depends on the density profile of the sphere. For consistency with our previous analysis, we choose to model the core as a uniform sphere of radius $R$, for which $\eta=3 / 5$.

\subsubsection{Magnetic Energy}

Magnetic energy is given, in SI units, by

$$
E_{B}=\frac{B^{2} V}{2 \mu_{0}},
$$

where $B$ is total magnetic field strength, and volume $V=$ $(4 \pi / 3) R^{3}$. We again take $B \approx B_{\text {pos }}$, noting that this will produce an underestimate of the true magnetic energy. If the statistical correction of Crutcher et al. (2004) applies to our results, the true magnetic energies will typically be $\sim 1.6 \times$ those that we derive. We give our values without correction, noting that they are, as with the other energies listed, accurate to order of magnitude.

\subsubsection{Kinetic Energy}

Thermal kinetic energy is given by

$$
E_{K, \mathrm{~T}}=\frac{3}{2} M \frac{k_{\mathrm{B}} T}{\mu m_{\mathrm{H}}},
$$

and nonthermal kinetic energy is given by

$$
E_{K, \mathrm{NT}}=\frac{1}{2} M \sigma_{\mathrm{NT}}^{2}
$$

For L1689N we again calculate these values both including and excluding the mass of IRAS 16293-2422.

\subsubsection{Rotational Energy}

Loren et al. (1990) inferred SMM-16 to be rotating with an angular velocity of $\omega=1.85 \pm 0.25 \mathrm{~km} \mathrm{~s}^{-1} \mathrm{pc}^{-1}$, with a rotation axis of $68^{\circ} \mathrm{E}$ of $\mathrm{N}$, using 2.4 arcmin resolution $\mathrm{DCO}^{+}$data, and so for SMM-16 we also calculate a value for
Table 3

Energetics of L1689N, SMM-16, and L1689B

\begin{tabular}{lcccr}
\hline \hline Energy $\left(\times 10^{35} \mathrm{~J}\right)$ & \multicolumn{2}{c}{ L1689N } & SMM-16 & L1689B \\
\cline { 2 - 4 } & All & With Masking & & \\
\hline Gravitational $\left(E_{G}\right)$ & -274 & -75 & -7.3 & -0.16 \\
Magnetic $\left(E_{B}\right)$ & $\ldots$ & 113 & 11 & 0.17 \\
Thermal kinetic $\left(E_{K, \mathrm{~T}}\right)$ & 18 & 9.5 & 2.2 & 0.25 \\
Nonthermal kinetic & 11 & 5.9 & 1.2 & 0.04 \\
$\quad\left(E_{K, \mathrm{NT}}\right)$ & $\ldots$ & & & $\ldots$ \\
Rotational $\left(E_{R}\right)$ & & $\ldots$ & 0.03 & $\ldots$ \\
\hline
\end{tabular}

rotational energy,

$$
E_{R}=\frac{1}{2} \mathcal{I} \omega^{2},
$$

where $\mathcal{I}$, the moment of inertia, is

$$
\mathcal{I}=\frac{2}{5} M R^{2}
$$

again taking SMM-16 to be a sphere of uniform density.

\subsubsection{Comparison of Values}

The energy values for each clump are listed in Table 3. We note that these values have significant uncertainties, and thus that the energy values that we derive are accurate only to order of magnitude. However, all of the derived energy values depend linearly on dust opacity $\kappa_{\nu}$, our dominant and systematic source of uncertainty, except for GPE, which varies as $\kappa_{\nu}^{2}$. Thus we consider only fiducial- $\kappa_{\nu}$ values here, because we can meaningfully compare different energy terms to one another despite the large uncertainties on their absolute values.

We note that the energy values that we list are largely a restatement of the mass-to-flux ratios and Alfvén Mach numbers previously calculated, with $E_{G} / E_{B} \propto \lambda^{2}$ and $E_{K, \mathrm{NT}} / E_{B} \propto \mathcal{M}_{A}^{2}$. Nonetheless, calculation of energy terms is a helpful exercise, allowing the various forces determining the evolution of the clump/core to be compared to one another in the same terms. We broadly expect any relatively long-lived object in the ISM to be near equipartition, and in L1689 we particularly expect gravitational and magnetic energies to to comparable, as all of our cores have mass-to-flux ratios consistent with unity.

We find that in L1689N, when IRAS 16293-2422 is excluded, the gravitational and magnetic energies are similar, with the former slightly smaller than the latter. When the mass of IRAS 16293-2422 is included, the GPE of L1689N is larger than, but still comparable to, the magnetic energy, consistent with the fragmentation across field lines and ongoing star formation in the clump, and with $\lambda>1$, which we infer in this case. In L1689N in particular, we note that a uniform sphere model is a crude approximation to the true geometry of the clump, which has fragmented into two significant cores, and we stress that the GPE values that we measure are approximate.

In SMM-16 and L1689B, the gravitational, magnetic, and kinetic energies are comparable to one another, as we expect. The rotational energy of SMM-16 is two orders of magnitude smaller than any of the other terms. SMM-16 and L1689B being in approximate equipartition is consistent with their lack of ongoing star formation. 


\section{Grain Alignment in L1689}

There is ongoing debate over the degree to which grains remain aligned relative to their local magnetic field direction at high $A_{V}$ (Whittet et al. 2008; Alves et al. 2014; Jones et al. 2015; Wang et al. 2019; Pattle et al. 2019). In the diffuse ISM, dust grains are expected to be aligned with their minor axes parallel to the local magnetic field direction (Davis \& Greenstein 1951). However, at high optical depths, grains are expected to become less effectively aligned with the magnetic field (e.g., Andersson et al. 2015). This is a corollary of the radiative alignment torques (RATs) model of grain alignment, in which irregularly-shaped grains are spun up by incident photons from a non-isotropic radiation field (Dolginov \& Mitrofanov 1976; Lazarian \& Hoang 2007). In this paradigm, high extinction will perforce lead to a lack of grain alignment.

\subsection{Measurements}

In order to confirm the validity of our preceding use of polarization observations to analyze the properties of the magnetic fields in L1689, we assess the degree of grain alignment in each clump. Grain alignment can be quantified through examination of the relationship between polarization efficiency and visual extinction (e.g., Whittet et al. 2008; Jones et al. 2015). In submillimeter emission polarimetry, this is commonly treated as a relationship between $p$ and $I$ (e.g., Alves et al. 2014). Polarization efficiency is identical to polarization fraction for optically thin emission (Alves et al. 2015), and for optically thin, isothermal dust emission, total intensity is proportional to visual extinction (Jones et al. 2015; Santos et al. 2017). Observations of polarized dust emission typically show a power-law dependence, $p \propto I^{-\alpha}$, where $0 \leqslant \alpha \leqslant 1$. A steeper index (higher $\alpha$ ) indicates poorer grain alignment: $\alpha=0$ indicates that grains are equally well aligned at all depths, while $\alpha=1$ indicates either a total absence of aligned grains or that all observed polarized emission is produced in a thin layer at the surface of the cloud (Pattle et al. 2019).

We apply the method of fitting the $p-I$ relationship described by Pattle et al. (2019) to our observations of L1689. The details of this analysis are described in Appendix C. We find that the fitting results for all regions agree with one another within error. We find $\alpha=0.55 \pm 0.03$ in L1689N, while we find $\alpha=0.59 \pm 0.06$ in SMM-16 and $\alpha=0.56 \pm 0.09$ in L1689B. These results are shown in Figure 7. This suggests that in all three regions grains become less well aligned with the magnetic field as density increases, but some degree of alignment persists to the highest densities that we observe, justifying our use of our polarization observations to analyze the properties of the magnetic fields that permeate the clumps.

\subsection{Comparison with $L 1688$}

The degree of grain alignment in L1689 appears to be broadly consistent with that recently measured in the neighboring L1688 region (Pattle et al. 2019). The $\alpha$ values observed in L1689 are larger than that of the externally illuminated Oph A region $(\alpha=0.34)$, but comparable to those of Oph B and C ( $\alpha \approx 0.6-0.7)$ (Pattle et al. 2019), and, in the cases of L1689N and SMM-16, better characterized than those in Oph B and C. The global interstellar radiation field on L1689 is likely to be similar to that on L1688, thanks to their comparable proximity to Sco OB2, as discussed in Section 2.
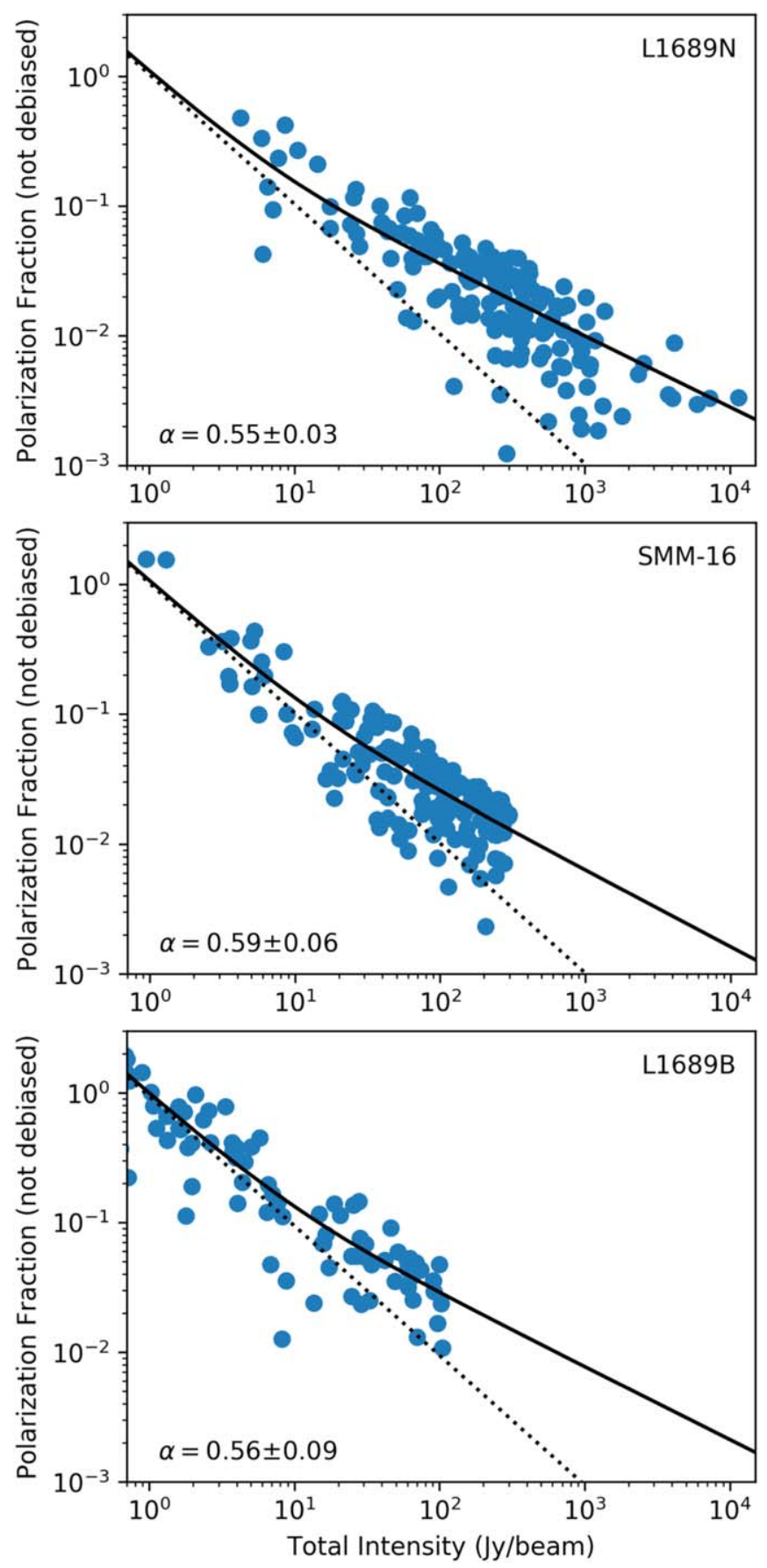

Figure 7. Polarization fraction $p$ (not debiased) vs. total intensity, fitted with the mean of the Ricean distribution of $p$ associated with a $p \propto I^{-\alpha}$ model (see Appendix $\mathrm{C}$ for details). The solid line shows best-fit model; the dashed line shows the expected behavior of nonaligned grains.

The agreement between the fitting results for L1689N and the starless sources suggests that the presence of the L1689N protostellar system is not significantly affecting the grain alignment in the clump as a whole, although it may be aligning grains on scales smaller than can be resolved by the JCMT. The only clump in Ophiuchus showing a significant difference in behavior is Oph A, which is illuminated by the two B stars of L1688, in which grains appear to be significantly better aligned with the magnetic field (Pattle et al. 2019). This provides further evidence suggesting that grain alignment within the 
dense clumps embedded within L1688 and L1689 is driven by the local radiation field on the clump.

\subsection{Grain Growth in L1689}

Our observations show that grains in L1689 retain some degree of alignment up to the highest gas densities that we observe. In RAT alignment theory, grains are efficiently aligned when they can be spun up to suprathermal rotation by an anisotropic radiation field, with there being a critical grain size $s_{\text {crit }}$ above which grains can become aligned, which increases with increasing gas density (Lazarian \& Hoang 2007; Hoang \& Lazarian 2008). We calculate this critical grain size for the clumps of L1689 using Equation (3) of Lee et al. (2020), assuming $T_{\mathrm{gas}}=T_{\text {dust }}=12 \mathrm{~K}$, and taking grain mass density $\rho=3 \mathrm{~g} \mathrm{~cm}^{-3}$, radiation field anisotropy $\gamma=0.1$ (Draine \& Weingartner 1996), and mean incident wavelength $\bar{\lambda}=1.2 \mu \mathrm{m}$ (Mathis et al. 1983; Draine et al. 2007). We further take the radiation strength (the ratio of local radiation energy density to that of the interstellar radiation field) to be $U=\left(T_{\text {dust }} / 16.4 \mathrm{~K}\right)^{6} \simeq 0.1535$ (Draine 2011). With these assumptions, and in the limit where hydrogen number density $n(\mathrm{H})=2 n\left(\mathrm{H}_{2}\right) \gg 30 \mathrm{~cm}^{-3}$, the critical grain size is given by

$$
s_{\text {crit }} \approx 3.88 \times 10^{-6} \mathrm{~cm} \times\left(\frac{n(\mathrm{H})}{30 \mathrm{~cm}^{-3}}\right)^{5 / 16} .
$$

Taking the range of $n(\mathrm{H})$ values corresponding to the average densities listed in Table 2, we find that in L1689N, for $n(\mathrm{H})=7 \times 10^{5} \mathrm{~cm}^{-3}, s_{\text {crit }} \approx 0.90 \mu \mathrm{m}$, while in the lowestdensity source, L1689B, for $n(\mathrm{H})=2.6 \times 10^{5} \mathrm{~cm}^{-3}$, $s_{\text {crit }}=$ $0.66 \mu \mathrm{m}$. We note that in Equation (18) we have conservatively adopted $\gamma=0.1$. Draine \& Weingartner (1996) found $\gamma=0.1$ in the diffuse ISM and $\gamma=0.7$ in molecular clouds, while Bethell et al. (2007) found $\gamma \sim 0.34$ in clumpy molecular clouds. If we take $\gamma=0.7$, our values of $s_{\text {crit }}$ will be smaller by a factor 0.55 , with $s_{\text {crit }, \gamma=0.7}=0.49 \mu \mathrm{m}$ and $0.36 \mu \mathrm{m}$ in L1689N and L1689B respectively. The maximum grain size in the diffuse ISM is $\sim 0.25-0.3 \mu \mathrm{m}$ (Mathis et al. 1977; Draine et al. 2007). Thus, as our observations show that a significant population of dust grains remain aligned at high densities in L1689, grain growth must have occurred. That such grain growth and evolution take place is suggested by recent studies of Herschel Space Observatory data, which show that dust opacity increases with column density in nearby molecular clouds (e.g., Roy et al. 2013; Ysard et al. 2013; Juvela et al. 2015). In particular, Schirmer et al. (2020) find that dust emission modeling of the Horsehead Nebula requires a minimum grain size $2-2.5 \times$ that in the diffuse ISM to reproduce Herschel and Spitzer observations. Polarization observations such as ours provide independent confirmation that grain growth occurs in these dense environments.

\section{Discussion}

L1689N, SMM-16, and L1689B all appear to be, on the scales probed by POL-2, magnetically transcritical environments with sub-Alfvénic turbulence. However, the three regions appear to have evolved in quite different manners.

Planck measurements (Planck Collaboration et al. 2015; Figure 2) show that in L1689 the magnetic field goes from running $\mathrm{NE} / \mathrm{SW}$ in the north, similar to the overall magnetic field direction across the complex $\left(\sim 50^{\circ} \mathrm{E}\right.$ of $\mathrm{N}$; Vrba et al. 1976), to running approximately $\mathrm{N} / \mathrm{S}$ in the south. In our data, we see agreement between POL-2 and Planck magnetic fields in L1689N and L1689B, but an approximately $80^{\circ}$ disagreement at the highest column densities in SMM-16, as shown in Figure 5.

\section{1. $L 1689 N$}

On cloud-to-clump scales, L1689N appears to be undergoing magnetically mediated evolution. The average magnetic field direction is uniform from Planck to JCMT scales. The magnetic field that we infer from our POL-2 observations is magnetically transcritical and uniform in the clump's center, albeit with some deviation from linearity in its NW and SE edges. Moreover, the clump itself is significantly elongated perpendicular to the plane-of-sky magnetic field direction, consistent with having formed through collection of material along magnetic field lines. L1689N thus, on clump scales, appears consistent with having formed in a magnetically dominated environment.

Despite the properties of the L1689N clump being broadly consistent with having formed in a strongly magnetized environment, the internal structure of the clump suggests that the magnetic field does not control the evolution of the starforming cores embedded within it. Our energetics analysis suggests that the clump is sufficiently massive to be gravitationally bound and unstable to fragmentation. The fragmentation across magnetic field lines within the L1689N clump, which has created the IRAS 16293-2422 and IRAS $16293 \mathrm{E}$ cores, and the ongoing star formation in the clump, indicated by the presence of the IRAS 16293-2422 protostellar system, confirm that the clump must be magnetically supercritical on some scales. Moreover, the magnetic field direction that we infer in IRAS 16293-2422 is perpendicular to that in the surrounding clump, indicating that the magnetic field direction is not consistent on all scales. Thus even if the magnetic field has been instrumental in forming the L1689N clump, as is suggested but not confirmed by our measurements, it appears not to be the dominant influence on the stars forming within it.

\subsubsection{IRAS 16293-2422}

The IRAS 16293-2422 system has been observed in $850 \mu \mathrm{m}$ polarized light using both the Submillimeter Array (SMA) (Rao et al. 2009) and ALMA (Sadavoy et al. 2018). The A and B protostars are linked by a "bridge" of emission oriented approximately SE/NW (e.g., Pineda et al. 2012; Jørgensen et al. 2016). The magnetic field in the IRAS 16293-2422 system runs along this bridge (Rao et al. 2009; Sadavoy et al. 2018 ), with a magnetic field strength of $23-78 \mathrm{mG}$, at a density of $5.6 \times 10^{8} \mathrm{~cm}^{-3}$ (Sadavoy et al. 2018).

In Figure 8 we compare the magnetic field direction that we infer on the position of IRAS 16293-2422 with that inferred by Rao et al. (2009) from SMA observations. In our POL-2 observations we see an average magnetic field direction across the core of $166^{\circ} \pm 31^{\circ}$, consistent with the SE/NW field seen in interferometric observations. The average field direction in the observations of Rao et al. (2009) is $152^{\circ} \pm 66^{\circ}$, although this value includes substantial contributions from the IRAS 16293A and B protostellar disks, polarized emission from which is likely to be dominated by scattering (Sadavoy et al. 2019). The 


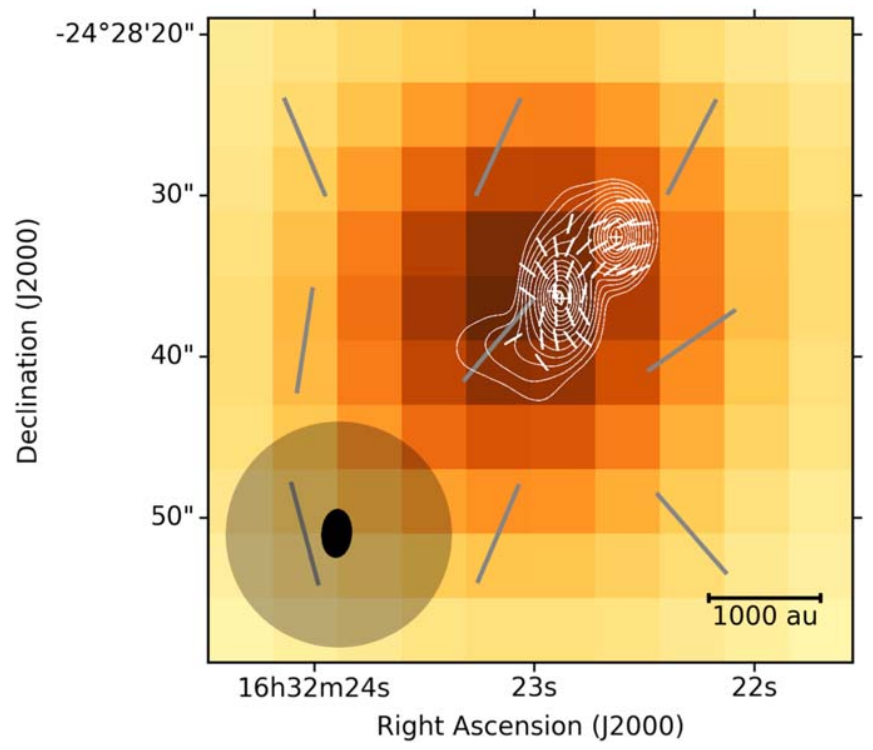

Figure 8. A comparison of POL-2 and SMA observations of IRAS 162932422. The underlying image is of POL-2 Stokes $I$ emission in which IRAS $16293-2422$ is a point source. Gray half-vectors show the POL-2 magnetic field direction. White contours show SMA Stokes $I$ emission, while white halfvectors show the SMA-inferred magnetic field direction (Rao et al. 2009). The JCMT and SMA beam sizes are shown in the lower left corner as a translucent gray circle and solid black ellipse respectively. Note that the registration of the two images is based on the nominal coordinates of each observation, and that the JCMT has a typical pointing uncertainty of $2^{\prime \prime}-6^{\prime \prime}\left(0.5-1.54^{\prime \prime}\right.$ pixels) (Mairs et al. 2017), comparable to or larger than the SMA beam.

average field direction in the observations of Sadavoy et al. (2019) is $176^{\circ} \pm 54^{\circ}$, again including both of the protostellar systems, while in the bridge feature the average value is $130^{\circ} \pm 14^{\circ}$. All of these values are consistent with our measurement.

If the dust emission features observed with ALMA formed from flux-frozen collapse under gravity, we can estimate what the magnetic field strength would have been in the gas from which the core formed. If we take $B=$ $(23-78 \mathrm{mG}) \times \sqrt{n /\left(5.6 \times 10^{8} \mathrm{~cm}^{-3}\right)}$ (assuming that in the high-density ISM, $B \propto n^{0.5}$; see Crutcher et al. 2010), then for our estimated density in the L1689N clump, $n=1.8 \times$ $10^{5} \mathrm{~cm}^{-3}$, we infer a field strength of $412-1398 \mu \mathrm{G}$, consistent with our inferred field strength of $160-580 \mu \mathrm{G}$. This suggests that the features observed on interferometric scales could have evolved from the larger-scale field that we observe.

Jacobsen et al. (2018) proposed a model of IRAS 162932422 in which material is accreting along the bridge of emission onto the two protostars. Sadavoy et al. (2018) posit that if the bridge is not a transient structure, it must itself be accreting material from the surrounding envelope, and so that the field in the surrounding envelope ought to be perpendicular to that in the bridge (see Gómez et al. 2018). This is what we see in our observations (Figure 4), supporting the suggestion that accretion onto the IRAS 16293-2422 protostars is magnetically regulated.

\subsubsection{IRAS $16293 E$}

As IRAS 16293E is a starless core, without a central hydrostatic object (Kirk et al. 2017), its geometry is not consistently defined between studies. Pattle et al. (2015) split the core into two components (their SMM-19 and SMM-22), the former approximately circular with an aspect ratio 1.1 , the latter with an aspect ratio of 1.6, oriented $44.7 \mathrm{E}$ of $\mathrm{N}$ (these sources are marked on Figure 3). Ladjelate et al. (2020) class IRAS 16293E as a single core (their source 464), with an aspect ratio 1.7 , oriented $2^{\circ} \mathrm{E}$ of N. Kirk et al. (2017), observing with ALMA, find an aspect ratio of 2.0 , oriented 4.8 $\mathrm{E}$ of $\mathrm{N}$ (their source 37 ). The core is thus consistently found to be elongated broadly $\mathrm{NE} / \mathrm{SW}$, in a direction similar to the local average magnetic field direction. This is not consistent with the behavior predicted for a strongly magnetized starless core (Fiedler \& Mouschovias 1993) or with the larger-scale behavior of the clump, again suggesting that the magnetic field does not control the evolution of the cores embedded within the L1689N clump.

\section{2. $S M M-16$}

The magnetic field that we infer from POL-2 data in SMM16 is, in the center of the clump, oriented approximately $90^{\circ} \mathrm{E}$ of $\mathrm{N}$, in contrast to the magnetic field at the same location inferred from the Planck data, which is oriented approximately $10^{\circ} \mathrm{E}$ of $\mathrm{N}$. This significant difference in magnetic field direction between cloud and clump scales suggests that a largescale reordering of the magnetic field has taken place during the formation of the clump.

Despite evidence for a large-scale rotation gradient across the region (Loren et al. 1990), our energetics analysis suggests that the rotational energy of SMM-16 itself is two orders of magnitude smaller than the gravitational, magnetic, and kinetic energies. This suggests that, whatever the cause of the reordering of the magnetic field in the clump, its dynamics are not currently significantly affected by rotation, as expected from previous studies of rotation in dense cores (Caselli et al. 2002; Tafalla et al. 2004; Xu et al. 2020). The similarity between POL-2 field lines in the periphery of the clump and the field direction inferred from Planck data suggests that the magnetic field in the clump may remain connected to the larger-scale field, despite its reordering in the center.

The density structure of the clump-in particular the multiple dense cores identified by Pattle et al. (2015) and Ladjelate et al. (2020)_and the complex velocity structure (Chitsazzadeh et al. 2014) suggest that the kinematics of the clump itself are more complex than that of simple solid-body rotation. Our energetics analysis suggests that the clump is not sufficiently dense to be undergoing gravitational fragmentation and collapse. If the sources identified by Pattle et al. (2015) and Ladjelate et al. (2020) represent distinct cores, rather than being transient features in an oscillating clump structure (Chitsazzadeh et al. 2014), they are unlikely to be gravitationally unstable.

In the dense center of SMM-16, the magnetic field appears to be strong enough to provide significant support against gravitational collapse. We posit that the misalignment between the magnetic field in the clump and that in the surrounding cloud means that, if material is accreted onto clumps along magnetic field lines, SMM-16 may not be able to efficiently acquire further mass from its surroundings, because even if the magnetic field in the clump does remain connected to the larger-scale field, infalling material would have to lose a significant amount of momentum in order to flow along the twisted magnetic field lines onto the core. SMM-16 may thus have been prevented from yet becoming sufficiently massive to form stars. 


\section{3. $L 1689 B$}

The magnetic field that we infer from POL-2 data in L1689B runs approximately $\mathrm{N} / \mathrm{S}$, perpendicular to the filament in which the core is embedded, and approximately parallel to the Planckscale magnetic field.

L1689B is a candidate gravitationally bound prestellar core. Its stability and age remain uncertain, with a number of studies identifying the core as contracting or showing signs of infall (Redman et al. 2004; Sohn et al. 2007; Lee \& Myers 2011; Seo et al. 2013), while Schnee et al. (2013) found it to be static. Redman et al. (2002) found L1689B to be relatively long-lived, with an age of at least one freefall time inferred from $\mathrm{CO}$ freezeout in the core's center; however, Lee et al. (2003) argued that the core is chemically young, with a lack of freezeout of $\mathrm{HCO}+$ and also a potential lack of $\mathrm{CO}$ freezeout. The transcritical mass-to-flux ratio that we measure suggests a dynamically important magnetic field in the core. While this does not provide information on the core's age, it does suggest magnetic support as a means by which the core could be longlived (e.g., Jessop \& Ward-Thompson 2000).

Redman et al. (2004) inferred a SW/NE $\left(\sim 45^{\circ} \mathrm{E}\right.$ of $\left.\mathrm{N}\right)$ rotation axis in L1689B, different from both the POL-2 and Planck magnetic field directions. This rotation axis is similar to the rotation axis in SMM-16, and the core is rotating in the same sense. Seo et al. (2013) found infall velocities in L1689B greater than could be caused by gravitational collapse, and so inferred that core collapse has been instigated by some sort of external perturbation, suggesting that turbulence or a sudden increase in external pressure might be responsible.

Our results suggest that the magnetic field is likely to be dynamically important in the evolution of the core, and that the core may be accreting material along magnetic field lines. Despite this, we do not see any suggestion of an hourglass field morphology in the core (see Fiedler \& Mouschovias 1993), but note that, due to the relatively low signal-to-noise ratio of our observations of L1689B, we do not detect polarization in the periphery of the core, where such a field structure would be most apparent.

\subsection{Magnetic Field Orientation with Respect to the L1689 Cloud and Its Substructures}

We next consider the orientation of the magnetic field with respect to the L1689 cloud, the filaments embedded within the cloud, and the clumps/cores that we observe.

\subsubsection{Cloud/Magnetic Field Alignment}

Molecular clouds typically have a large-scale magnetic field direction that is either parallel or perpendicular to the major axis of the molecular cloud ( $\mathrm{Li}$ et al. 2013). In both near-IR and Planck observations, the magnetic field of L1689 is parallel to the major axis of the cloud (see Figures 1 and 2; Li et al. 2013; Planck Collaboration et al. 2015). Li et al. (2017) further suggest that clouds that formed parallel to their magnetic field direction have higher star formation rates, because the field does not hinder gravitational fragmentation, taking Ophiuchus as an example of a parallel-field cloud.

Planck observations of Ophiuchus have found that at lower column densities the magnetic field is parallel to density structures, with some hint of a transition to perpendicularity at high column densities (Planck Collaboration et al. 2016). Soler (2019) identified this transition as occurring at $N(\mathrm{H}) \sim 10^{21.8} \mathrm{~cm}^{-2}$ in the L1688/L1689 region. However, this study did not distinguish between L1688 and L1689, and we note that the mass distribution of Ophiuchus is dominated by L1688, which has approximately 2.5 times the mass of L1689 (Loren 1989). While L1688 has a clearly defined major axis (e.g., Ladjelate et al. 2020), L1689 has a more complex geometry, and its preferred orientation with respect to the large-scale magnetic field is less well defined. Planck Collaboration et al. (2016) identify an average magnetic field strength in the Ophiuchus molecular cloud of $B_{\text {pos }} \sim 13-25 \mu \mathrm{G}$, and a mass-to-flux ratio $\lambda \sim 0.2-0.4$, suggesting that on large scales the cloud is significantly magnetically subcritical. We note, however, that interpretation of the large-scale magnetic field in Ophiuchus is complicated by feedback effects from the Sco OB2 association. The large-scale dust emission and magnetic field structures of L1689 in particular are clearly bowed, indicative of the large-scale W-to-E influence on the cloud (Figures 1 and 2, Vrba et al. 1976; Loren 1989).

\subsubsection{Filament/Magnetic Field Alignment}

Herschel observations (Arzoumanian et al. 2019; Ladjelate et al. 2020) have shown filaments in Ophiuchus to be preferentially aligned either parallel or perpendicular to the large-scale NE/SW streamers (Loren 1989), and so to the large-scale magnetic field direction (Vrba et al. 1976; Planck Collaboration et al. 2015; see Figure 1). Figure 2 shows that the filaments in which L1689N and L1689B are embedded are part of, and approximately parallel to the overall direction of, the L1689/L1712 filamentary streamer and perpendicular to the Planck-scale magnetic field direction, while SMM-16 is embedded in a filament that is approximately parallel to the local Planck-scale field and perpendicular to the major axis of the L1689/L1712 filamentary streamer.

\subsubsection{Filament Stability}

Arzoumanian et al. (2019) produced a catalog of filamentary structures across all regions observed by the Herschel Gould Belt Survey (André et al. 2010). We took their values for the masses per unit length and temperatures of the principal filaments on which our three clumps are located. Arzoumanian et al. (2019) find three principal filaments meeting in L1689N; two, running approximately $\mathrm{N}-\mathrm{S}$, meeting in SMM-16; and one running approximately $\mathrm{E}-\mathrm{W}$ through L1689B. ${ }^{33}$ These filaments are very similar to those found by Ladjelate et al. (2020) in the same region, shown in Figure 2.

The critical mass per unit length according to Ostriker (1964) for a uniform, unmagnetized, isothermal filament is given by

$$
\left(\frac{M}{L}\right)_{\text {crit }}=\frac{2 c_{s}^{2}}{G}
$$

where $c_{s}$ is the sound speed in the filament, $c_{s}=\left(k_{\mathrm{B}} T / \mu m_{\mathrm{H}}\right)^{0.5}$. Arzoumanian et al. (2019) give integrated line masses $(M / L)$ of 12.7, 20.6, and $36.8 M_{\odot} \mathrm{pc}^{-1}$ and temperatures of 16.1, 15.1, and $15.8 \mathrm{~K}$ for the three filaments in L1689N. Neglecting any contribution from nonthermal sources of support, these values result in critical line mass ratios $(M / L) /(M / L)_{\text {crit }}$ of 0.57 , 1.00 , and 1.70 , respectively. Similarly, the two filaments that

\footnotetext{
33 L1689N filament indices: 8, 10, 35; SMM-16 filament indices: 29, 54; L1689B filament index: 6, in the nomenclature of Arzoumanian et al. (2019). Filament skeleton maps are available from the Herschel Gould Belt Survey archive at http://www.herschel.fr/cea/gouldbelt/en/.
} 
meet at SMM-16 have integrated line masses of 5.5 and 15.7 $M_{\odot} \mathrm{pc}^{-1}$, and temperatures of 15.8 and $16.2 \mathrm{~K}$, resulting in thermal critical line masses of 0.25 and 0.72 , respectively. Finally, the filament containing L1689B has an integrated line mass of $19.1 M_{\odot} \mathrm{pc}^{-1}$ and a temperature of $15.5 \mathrm{~K}$, and so a critical line mass ratio of 0.90 . If we instead adopt a temperature of $12 \mathrm{~K}$ for the filaments, consistent with the temperature that we assume in the clumps/cores, these ratios increase by a factor 2.2. Arzoumanian et al. (2019) consider filaments with $0.5<(M / L) /(M / L)_{\text {crit }}<2$ to be marginally gravitationally unstable. Thus, even in the absence of magnetic or turbulent support, the filaments of L1689 are only marginally unstable. Despite the demonstrable gravitational instability of L1689N, the marginal stability of SMM-16, and signs of infall in L1689B (e.g., Lee et al. 2001), all three clumps appear to have formed within, or at the junction of, filaments that are not themselves significantly unstable, perhaps suggesting that the fragmentation of the filaments is driven by turbulent processes (e.g., Clarke et al. 2016). Gravitationally bound cores have previously been found in filaments with $(M / L)<(M / L)_{\text {crit }}$ in the California molecular cloud (Zhang et al. 2020), indicating that such a scenario is not unique to L1689.

\subsubsection{Magnetic Field Alignment within Clumps and Cores}

In all three of the clumps/cores in L1689 that we observe, the mean plane-of-sky magnetic field direction observed with POL-2 is significantly offset from the plane-of-sky major axis of the filament in which the clump/core is embedded (see Figure 5).

A number of recent theoretical and numerical studies have predicted that magnetic fields should turn to become perpendicular to magnetically supercritical filaments (see Hennebelle \& Inutsuka 2019, for a recent review). Seifried et al. (2020) find that magnetic fields that are initially parallel to filamentary structures at low densities become perpendicular at high densities, for initial magnetic field strengths $>5 \mu \mathrm{G}$. They identify this transition as occurring at $n \sim 10^{2}-10^{3} \mathrm{~cm}^{-3}$ (or $N \sim 10^{21}-10^{21.5} \mathrm{~cm}^{-2}$ ) and at $\lambda \sim 1$, with the mass distribution being magnetically subcritical on large scales, and supercritical in the dense material. This change of orientation is taken to be indicative of compressive motions, resulting from either converging flows or gravitational collapse, and of an initially dynamically important magnetic field that suppresses turbulent motions and promotes accretion of material and gravitational collapse (Soler \& Hennebelle 2017; Seifried et al. 2020). This picture is broadly consistent with what we see in L1689: a cloud-parallel magnetic field threading a magnetically subcritical mass distribution at low column densities (Planck Collaboration et al. 2016), which becomes approximately perpendicular to the high-column-density filaments in regions of potential gravitational instability. However, it should be noted that while models predict supercritical mass-to-flux ratios in filaments with perpendicular fields, we infer transcritical mass-to-flux ratios in all our clumps, despite their having densities $\gtrsim 10^{5} \mathrm{~cm}^{-3}$.

The behavior that we see in L1689 is consistent with other recent BISTRO survey observations of nearby star-forming regions, including Orion A (Ward-Thompson et al. 2017; Pattle et al. 2017), IC5146 (Wang et al. 2019) and NGC 1333
(Doi et al. 2020). Magnetic fields have also been observed to be perpendicular to filaments in POL-2 observations of more distant massive infrared dark clouds, including G34.43+0.24 (Soam et al. 2019; see also Tang et al. 2019) and G035.3900.33 (Liu et al. 2019). However, the proximity of L1689 allows us to examine the transition from cloud-parallel to filament-perpendicular fields at higher physical resolution than is possible elsewhere.

\subsection{Identifying the Transition from Sub- to Supercritical Dynamics}

The fact that filament directions in Ophiuchus are preferentially either parallel or perpendicular to the cloud-scale magnetic field direction, along with their lack of significant gravitational instability, suggests that the magnetic field may be dynamically important in filament formation. However, the magnetic field direction in the dense clumps and cores that we observe appears not to be consistently set by the cloud-scale magnetic field direction, suggesting that a transition from magnetically subcritical to supercritical dynamics may occur at size scales between those resolved by Planck and those observable with POL-2.

Although the direction of magnetic fields in our regions appears not to be determined by the cloud-scale field direction, the relative orientation of the cloud- and clump-scale fields may still influence their evolution. The apparent similarity between cloud- and clump-scale magnetic field directions in L1689N and L1689B (if not a projection effect) suggests that material can accrete onto these regions along magnetic field lines more efficiently than is the case in SMM-16.

A key unanswered question is whether the magnetic field direction in the filaments in which the clumps/cores are embedded is perpendicular to the filament, parallel to it, or inherited from the cloud-scale magnetic field direction. At present, the best tool available for examining magnetic field behavior in such intermediate-scale low-surface-brightness structures and identifying the column density at which the transition from supercritical to subcritical dynamics occurs is high-resolution near-IR extinction polarimetry (e.g., Kwon et al. 2015).

\section{Summary}

In this paper we have presented JCMT POL-2 observations of the L1689 molecular cloud in Ophiuchus, specifically of the star-forming clump L1689N, the starless clump SMM-16, and the starless core L1689B. Our key results are:

1. We noted that revised distance estimates to the L1688 and L1689 molecular clouds suggest that the two clouds are located at approximately equal distances from the Sco OB2 association, feedback from which is thought to strongly influence the evolution of both clouds.

2. L1689N has a linear magnetic field running approximately $\mathrm{NE} / \mathrm{SW}$, except at the position of the IRAS 16293-2422 protostellar system. The NE/SW field shows some signs of deviation from linearity in the periphery of the clump, but is on average approximately perpendicular to the major axis of the clump, and to the local direction of the L1689/L1712 filamentary streamer, as identified in Herschel observations. L1689N shows good agreement between magnetic field directions observed with Planck 
and with POL-2. SMM-16 has an approximately linear magnetic field running $\mathrm{E} / \mathrm{W}$ in its center, which curves toward running $\mathrm{N} / \mathrm{S}$ in its periphery. The central $\mathrm{E} / \mathrm{W}$ field is approximately perpendicular both to the field observed by Planck and to the filament in which SMM-16 is embedded. L1689B has a linear magnetic field running $\mathrm{N} / \mathrm{S}$, approximately parallel to the magnetic field direction observed with Planck, and perpendicular to the major axis of the L1689/L1712 filamentary streamer, in which it is embedded.

3. L1689N has a plane-of-sky magnetic field strength $B_{\text {pos }}=366 \pm 209 \mu \mathrm{G}$, a transcritical mass-to-flux ratio $\lambda=0.79 \pm 0.45(\lambda=1.52 \pm 0.86$ including the mass of the IRAS 16293-2433 system), and sub-Alfvénic nonthermal motions with an Alfvén Mach number $\mathcal{M}_{A}=0.23 \pm$ 0.05. SMM-16 has a field strength $B_{\text {pos }}=284 \pm 127 \mu \mathrm{G}$, a magnetically transcritical mass-to-flux ratio $\lambda=0.81 \pm$ 0.34 , and sub-Alfvénic nonthermal motions with $\mathcal{M}_{A}=$ $0.34 \pm 0.06$. L1689B has a field strength $B_{\text {pos }}=72 \pm$ $60 \mu \mathrm{G}$, a transcritical mass-to-flux ratio $\lambda=0.95 \pm 0.81$, and sub-Alfvénic nonthermal motions with $\mathcal{M}_{A}=0.47 \pm$ 0.26 . The uncertainties on $B_{\text {pos }}$ and $\lambda$ are dominated by systematic uncertainty on dust opacity; their statistical uncertainties are $15 \%, 11 \%$, and $46 \%$ on L1689N, SMM16, and L1689B respectively.

4. We found that L1689N is sufficiently massive to be unstable to gravitational fragmentation and collapse only when the mass of the IRAS 16293-24322 protostellar system is accounted for, and is otherwise energetically in approximate equipartition. In SMM-16 and L1689B the gravitational, magnetic, and kinetic energies are comparable to one another.

5. We found that dust grains in all three regions retain some degree of alignment with respect to the magnetic field at high column densities, suggesting that grain growth has occurred. Grains appear to be similarly well aligned in each region despite their differing star formation histories.

6. In all three regions, the plane-of-sky magnetic field direction is, in the clumps' centers, approximately perpendicular to the plane-of-sky major axis of the filament in which the clump is embedded, appearing to be set by the direction of the filament rather than by the large-scale magnetic field direction, in keeping with predictions from recent numerical modeling. However, the filaments in which the clumps are embedded are not themselves significantly gravitationally unstable, and appear to have formed in a magnetically subcritical environment. This suggests that a transition from magnetically subcritical to supercritical gas dynamics may occur on size scales between those resolved by Planck and those that we observe with POL-2.

7. We propose that star formation in L1689N is, on large scales, magnetically regulated, with consistent magnetic field morphology from cloud to clump scales, and a clump major axis perpendicular to the magnetic field direction. The magnetic field configuration may allow unrestricted flow of material onto L1689N along magnetic field lines, potentially provoking gravitational collapse. However, the fragmentation of the clump perpendicular to the large-scale field direction and the misalignment of the magnetic field in the IRAS 16293-
2422 protostar with that of the larger clump suggest that the region is not magnetically regulated on all size scales. Conversely, in SMM-16, the misalignment between the magnetic field in the clump and that in the surrounding cloud may prevent the clump from efficiently acquiring additional mass from its surroundings, potentially inhibiting gravitational collapse.

K.P. and S.P.L. acknowledge support from the Ministry of Science and Technology (Taiwan) under grant No. 106-2119M-007-021-MY3. S.P.L. further acknowledges support from the Ministry of Science and Technology (Taiwan) under grant No. 109-2112-M-007-010-MY3. J.D.F. and D.J. are supported by the National Research Council of Canada and by Natural Sciences and Engineering Research Council of Canada (NSERC) Discovery Grants. T.H. acknowledges the support from the National Research Foundation of Korea (NRF) grants funded by the Korea government (MSIT) through the Midcareer Research Program (2019R1A2C1087045). D.A. acknowledges support by FCT/MCTES through national funds (PIDDAC) by the grants UID/FIS/04434/2019 \& UIDB/ $04434 / 2020$. Y.D. was supported by Grants-in-Aid for Scientific Research (18H01250) from the Japan Society for the Promotion of Science (JSPS). L.F. acknowledges support from the Ministry of Science and Technology (Taiwan) under grant No. 107-2119-M-001-031-MY3 and from Academia Sinica under grant No. AS-IA-106-M03. C.L.H.H. acknowledges the support of the NAOJ Fellowship as well as JSPS KAKENHI grants $18 \mathrm{~K} 13586$ and $20 \mathrm{~K} 14527$. J.K. is supported by JSPS KAKENHI grant No. 19K14775. W.K. was supported by the New Faculty Startup Fund from Seoul National University and by the Basic Science Research Program through the NRF (NRF-2016R1C1B2013642). C.W.L. is supported by the Basic Science Research Program through the NRF funded by the Ministry of Education, Science and Technology (NRF2019R1A2C1010851). T.L. acknowledges support from the international partnership program of the Chinese Academy of Sciences through grant No.114231KYSB20200009 and the support from the National Natural Science Foundation of China (NSFC) through grant NSFC No. 12073061. A.S. acknowledges support from the National Science Foundation (NSF) through grant AST-1715876. M. Tamura is supported by JSPS KAKENHI grant Nos. 18H05442, 15H02063, and 22000005. This research is partially supported by Grants-in-Aid for Scientific Researches from the Japan Society for Promotion of Science (KAKENHI 19H0193810). The James Clerk Maxwell Telescope is operated by the East Asian Observatory on behalf of The National Astronomical Observatory of Japan; Academia Sinica Institute of Astronomy and Astrophysics; the Korea Astronomy and Space Science Institute; Center for Astronomical Mega-Science (as well as the National Key R\&D Program of China with No. 2017YFA0402700). Additional funding support is provided by the Science and Technology Facilities Council of the United Kingdom and participating universities in the United Kingdom, Canada, and Ireland. The authors wish to recognize and acknowledge the very significant cultural role and reverence that the summit of Maunakea has always had within the indigenous Hawaiian community. We are most fortunate to have the opportunity to conduct observations from this mountain.

Facility: James Clerk Maxwell Telescope (JCMT). 
Software: Starlink (Currie et al. 2014), Astropy (Astropy Collaboration et al. 2013, 2018), Simbad (Wenger et al. 2000).

\section{Appendix A}

\section{Polarization Properties}

The formulae used to determined derived polarization proerties are as follows: polarized intensity is given by

$$
P=\sqrt{Q^{2}+U^{2}},
$$

and debiased polarized intensity,

$$
P_{\mathrm{db}}=\sqrt{Q^{2}+U^{2}-\frac{1}{2}\left(V_{Q}+V_{U}\right)},
$$

where $V_{Q}$ is the variance of $Q$ and $V_{U}$ is the variance of $U$. We also take uncertainties on $Q, U$, and $I$ to be $\delta Q=\sqrt{V_{Q}}$, $\delta U=\sqrt{V_{U}}$, and $\delta I=\sqrt{V_{I}}$, respectively. Polarization fraction is then given by

$$
p=\frac{P}{I},
$$

and debiased polarization fraction by

$$
p_{\mathrm{db}}=\frac{P_{\mathrm{db}}}{I} .
$$

Uncertainties on polarization fraction (both debiased and nondebiased) are given by

$$
\delta p=\sqrt{\frac{Q^{2} \delta Q^{2}+U^{2} \delta U^{2}}{I^{2}\left(Q^{2}+U^{2}\right)}+\frac{\delta I^{2}\left(Q^{2}+U^{2}\right)}{I^{4}}} .
$$

Polarization angle is given by

$$
\theta_{p}=\frac{1}{2} \arctan \left(\frac{U}{Q}\right),
$$

and uncertainty on polarization angle by

$$
\delta \theta_{p}=\frac{1}{2} \frac{\sqrt{Q^{2} \delta U^{2}+U^{2} \delta Q^{2}}}{Q^{2}+U^{2}} .
$$

\section{Appendix B \\ Davis-Chandrasekhar-Fermi Analysis}

In this appendix we describe how we arrived at the values of gas density, magnetic field angle dispersion, and nonthermal velocity dispersion that we used in the DCF analysis described in Section 4.

\section{B.1. Gas Density}

We estimated the size of each of the clumps by fitting a 2D Gaussian distribution to the emission associated with the clump. In the case of L1689N, we masked emission in a the $40^{\prime \prime}$ diameter region surrounding the IRAS 16293-2422 protostar, as we aim to investigate the magnetic field strength in the larger clump, rather than the behavior of IRAS 16293-2422 itself, the magnetic field of which appears to be behaving quite differently to that in the clump in which it is embedded. The best-fit Gaussian distributions are listed in Table 1. The contours of 1 FWHM of the fitted ellipses are shown in Figure 9.

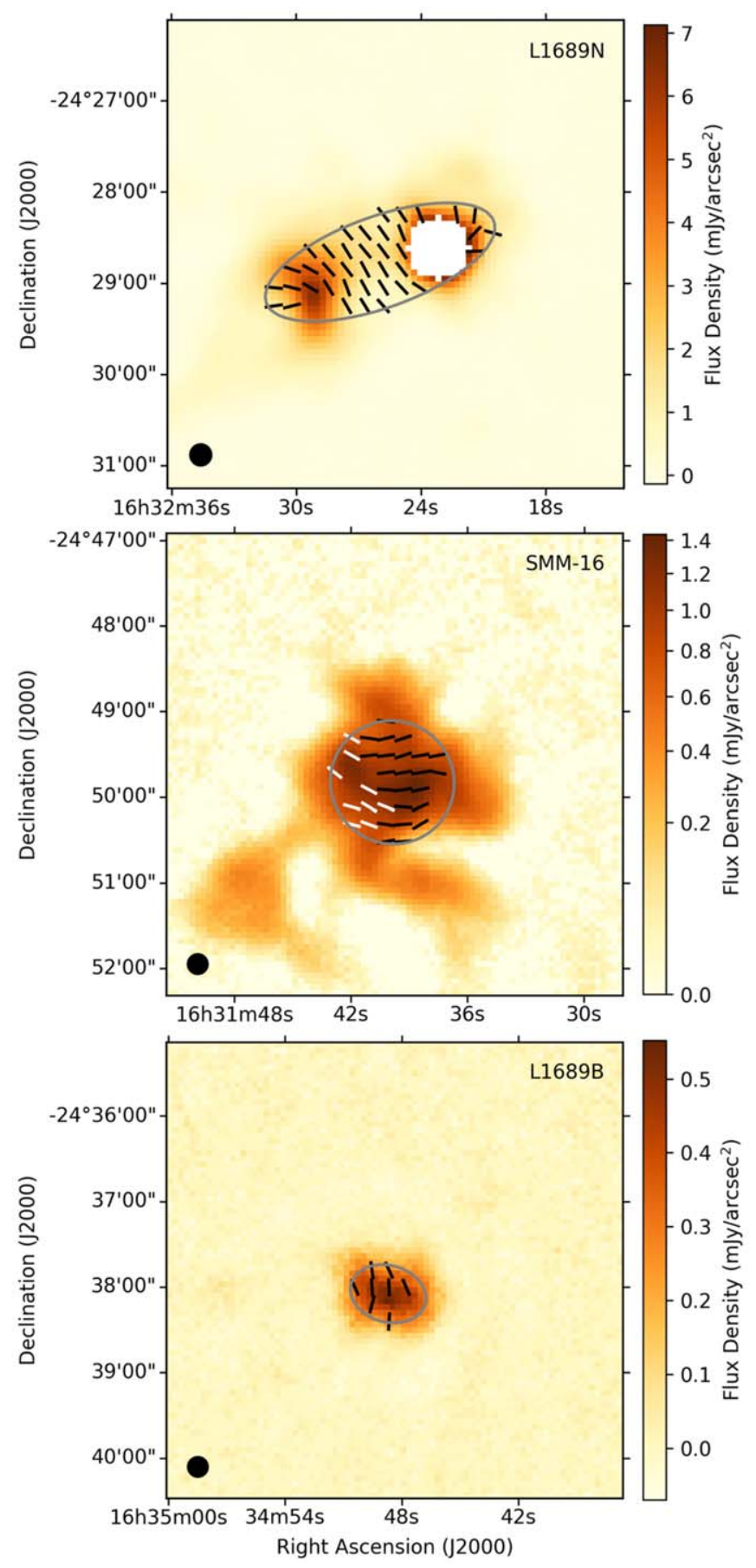

Figure 9. Areas over which magnetic field strengths were estimated, enclosed by contours of 1 FWHM of ellipses fitted to Stokes $I$ emission, in L1689N (top), SMM-16 (middle), and L1689B (bottom). Masked pixels in L1689N cover the IRAS 16293-2422 protostellar system. White half-vectors in SMM16 indicate the area of ordered deviation identified as the "East" Gaussian component.

We calculated gas mass $M\left(F_{\nu}, D, \kappa_{\nu}, T\right)$ using the relation of Hildebrand (1983) (Equation (8)). We determined $F_{\nu}$ by summing all emission within the aperture defined by the ellipse of 1 FWHM diameter for each clump, as defined in Table 1. We filled the masked pixels covering IRAS 16293-2422 with the mean flux density in the pixels bordering the masked region $\left(0.0793 \mathrm{Jy} \mathrm{pixel}^{-1}\right)$. We took $D=144.2 \mathrm{pc}$ (Ortiz-León et al. 2018) and $\kappa_{\nu}=0.0125 \mathrm{~cm}^{2} \mathrm{~g}^{-1}$ (e.g., Johnstone et al. 2017). We estimated gas temperatures in L1689N and SMM-16 by 
averaging the dust temperatures determined from spectral energy distribution fitting of Herschel and SCUBA-2 observations in each region by Pattle et al. (2015), assuming that the gas and dust are well coupled. In L1689N we averaged the temperatures of their sources SMM-19, -22, -23, -24, -25, and -26 (excluding SMM-20, their identifier for IRAS 16293-2422) to get a mean temperature of $11.7 \pm 1.4 \mathrm{~K}$. In SMM-16 we averaged the temperatures of their sources SMM-16a, -16b, and -16 to get a mean temperature of $12.2 \pm 0.4 \mathrm{~K}$. Thus for simplicity we took $T=12 \mathrm{~K}$ in both clumps, with uncertainties as given above. We also took $T=12 \mathrm{~K}$ for L1689B, following Redman et al. (2002).

The uncertainty on our mass estimates is dominated by the systematic uncertainty on dust opacity. We conservatively took our value of $\kappa_{\nu}$ to be accurate to $\sim 50 \%$ (Roy et al. 2014). We note that this results in a significantly larger mass uncertainty than is usual; however, we adopt this value in order to demonstrate the plausible range of magnetic field values associated with our measurements. We further took the uncertainty on $F_{\nu}$ to be dominated by the SCUBA-2 $850 \mu \mathrm{m}$ calibration uncertainty of $10 \%$ (Dempsey et al. 2013), and assumed a representative uncertainty of $\pm 1.5 \mathrm{~K}$ on $T$. We defined a representative source size $R$ as described in Equation (7) and thus calculated average column and volume densities using Equations (9) and (10) respectively. The flux densities, masses, and column and volume densities determined for each clump are listed in Table 2.

\section{B.2. Angle Dispersion}

The dispersion in angle $\sigma_{\theta}$ in the DCF relation measures intrinsic dispersion caused by Alfvénic distortion of the magnetic field by nonthermal motions. However, the observed distribution of magnetic field angles also contains contributions from large-scale field structure and from measurement uncertainties. Various methods have been proposed to account for ordered variation in magnetic field (see Pattle \& Fissel 2019 for a recent review). Here, we choose to measure the dispersion in magnetic field position angle by fitting a Gaussian distribution to the distributions of position angles. This method is suitable when a clearly identifiable and dominant linear magnetic field component exists-here, the NE/SW component in L1689N, the E/W component in SMM-16, and the $\mathrm{N} / \mathrm{S}$ component in L1689B.

We selected half-vectors where $p_{\mathrm{db}} / \delta p>3, I / \delta I>5$, and $\delta \theta<10^{\circ}$. For self-consistency, we restricted our sample to those half-vectors contained within 1 FWHM of the fitted Gaussian over which we estimated volume density. In L1689N, we excluded half-vectors coincident with the masked region around the position of IRAS 16293-2422. The half-vectors included in our DCF analysis are shown in Figure 9.

For L1689N and SMM-16, we fitted the histogram of position angles with a Gaussian function, as shown in Figure 10. The standard deviation of the fitted Gaussian was then taken to be the dispersion in angle $\sigma_{\theta}$. We found that L1689N was well fitted by a single Gaussian, with $\sigma_{\theta}=6.6 \pm 0.6$ and a mean value of $\langle\theta\rangle=34.2 \pm 0.8$. As SMM-16 shows ordered variation on the eastern side of the clump, we fitted both single- and double-Gaussian models. We found $\sigma_{\theta}=18.2 \pm 6.7\left(\langle\theta\rangle=90^{\circ} .6 \pm 2.6\right)$ for the singleGaussian model, whereas we found $\sigma_{\theta}=9^{\circ} .7 \pm 0.1 \quad(\langle\theta\rangle=$ $\left.95^{\circ} .9 \pm 0.4\right)$ for the central component of the double-Gaussian model and $\sigma_{\theta}=7.5 \pm 0.5 \quad\left(\langle\theta\rangle=65^{\circ} .2 \pm 0.7\right)$ for the
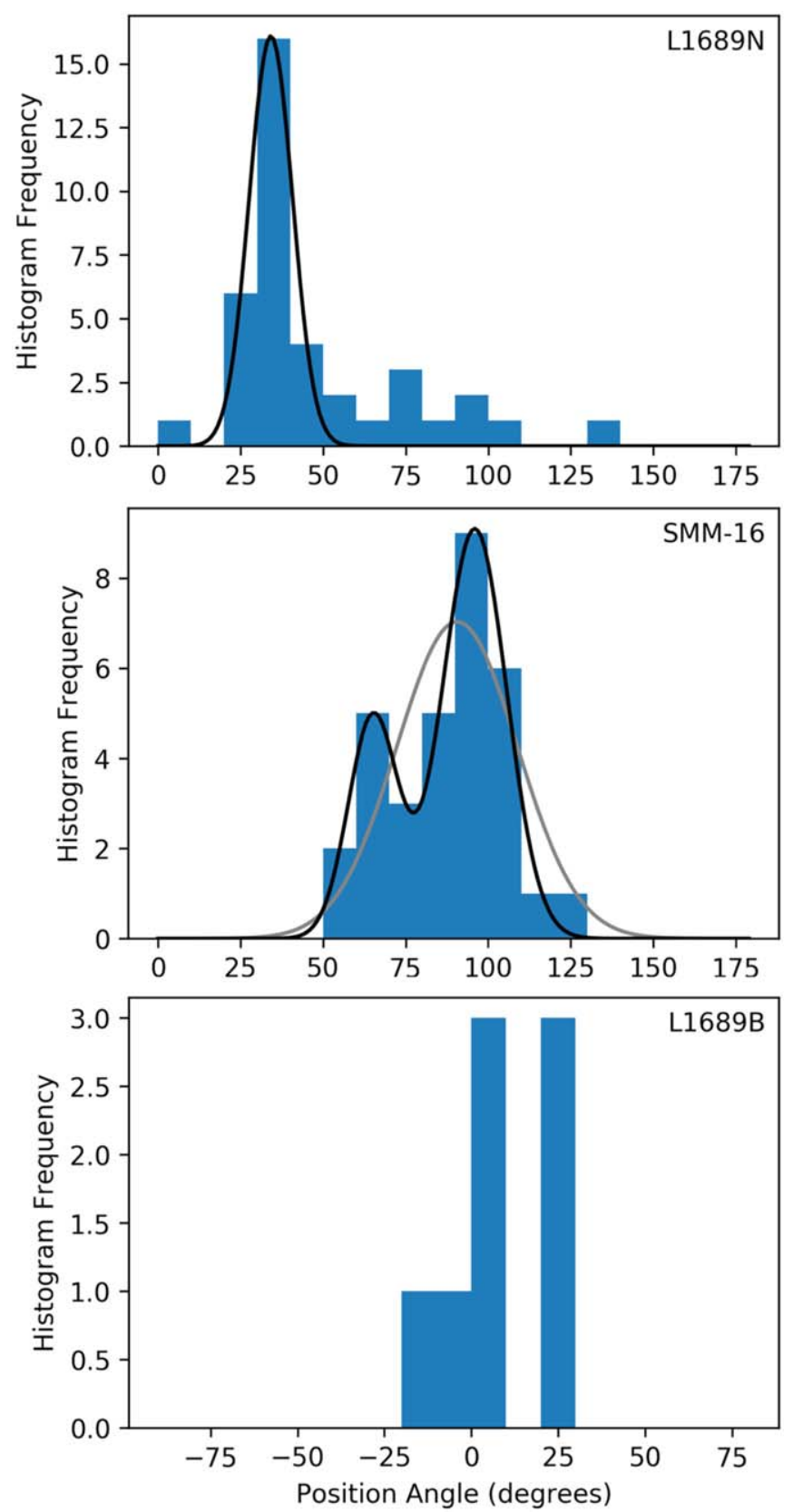

Figure 10. Histograms of magnetic field angle in L1689N (top), SMM-16 (middle), and L1689B (bottom), within areas enclosed by fitted ellipses. Position angles are in degrees $\mathrm{E}$ of $\mathrm{N}$, in the range $0^{\circ} \leqslant \theta<180^{\circ}$ for $\mathrm{L} 1689 \mathrm{~N}$ and SMM-16, and in the range $-90^{\circ} \leqslant \theta<90^{\circ}$ for L1689B. In L1689N, the best-fit Gaussian model is shown in black. In SMM-16, the best-fit singleGaussian model is shown in gray, and the best-fit double-Gaussian model is shown in black. L1689B does not have enough half-vectors to accurately fit a Gaussian model.

eastern component. The half-vectors covered by the two Gaussian components are marked on Figure 9, where black half-vectors indicate the central component. As the two Gaussian components are spatially distinct, we consider the two-Gaussian fit to produce dispersion values more representative of the underlying dispersion in angle, and so take $\sigma_{\theta}=9.7 \pm 0.1$ for SMM-16. L1689B does not have enough well characterized half-vectors to accurately fit a Gaussian model, and so we instead list the mean and standard deviation of the position angles of the half-vectors shown in Figure 9 in Table 2. We took the uncertainty on the measured standard 
deviation to be $\sigma_{\theta} / \sqrt{N}$, where $N$ is the number of half-vectors included, and so find $\sigma_{\theta}=13.3 \pm 4.7$.

The effect of measurement uncertainty on measured angular dispersion is small while measurement uncertainties are less than or similar to the intrinsic dispersion (Pattle et al. 2017). We further mitigated the effect of measurement uncertainties by choosing histogram bins of $10^{\circ}$, larger than the maximum allowed uncertainty in angle. In L1689N, the mean measurement uncertainty was $3^{\circ} .4$, the median $3{ }^{\circ} .1$, and the maximum $8^{\circ} .6$, suggesting that our measured dispersion $\sigma_{\theta}=6.6 \pm 0.6$ does not need to be corrected for the effects of measurement uncertainty. Similarly, in SMM-16, the mean measurement uncertainty was $5^{\circ} .8$, the median 5.5 , and the maximum $9^{\circ} .7$, again suggesting that our measured dispersion, $\sigma_{\theta}=9^{\circ} .7 \pm 0^{\circ} .1$, does not require correction.

\section{B.3. Gas Velocity Dispersion}

We took gas velocity dispersions for L1689N and SMM-16 from $\mathrm{N}_{2} \mathrm{H}^{+} J=1 \rightarrow 0$ measurements made by Pan et al. (2017) using the Purple Mountain Observatory Delingha $13.7 \mathrm{~m}$ telescope, with a beam size of $\sim 60^{\prime \prime}$ at $3.22 \mathrm{~mm}$. $\mathrm{N}_{2} \mathrm{H}^{+}$is a tracer of dense gas with a critical density of $10^{5}-10^{6}$ $\mathrm{cm}^{-3}$ (Pan et al. 2017), consistent with the mean volume densities in our clumps. We choose these measurements to ensure that both clumps are measured self-consistently, and because the PMO Delingha beam size is comparable to the size of the clumps, suggesting that the linewidths will be representative of the dense gas in each clump. Pan et al. (2017) list FWHM linewidths of $\Delta v=0.62 \pm 0.04 \mathrm{~km} \mathrm{~s}^{-1}$ and $\Delta v=0.59 \pm 0.04 \mathrm{~km} \mathrm{~s}^{-1}$ in L1689N and SMM-16 respectively (L1689NW and L1689W in their nomenclature).

We took the gas velocity dispersion of L1689B from $\mathrm{N}_{2} \mathrm{H}^{+}$ $J=1 \rightarrow 0$ measurements made by Lee et al. (2001) using the FCRAO $14 \mathrm{~m}$ telescope, with a beam size of $\sim 52^{\prime \prime}$. Lee et al. (2001) list an FWHM linewidth of $\Delta v=0.32 \pm 0.03 \mathrm{~km} \mathrm{~s}^{-1}$ for the source, averaged over eight pointings.

We estimated the nonthermal component of these linewidths using the relation

$$
\Delta v_{\mathrm{NT}}=\sqrt{(\Delta v)^{2}-8 \ln 2 \frac{k_{\mathrm{B}} T}{29 m_{\mathrm{H}}}},
$$

continuing to take $T=12 \mathrm{~K}$ in each clump, and noting that $\mathrm{N}_{2} \mathrm{H}^{+}$has a mass of $29 m_{\mathrm{H}}$. The correction for the thermal linewidth component is small. We assume that the nonthermal velocity component represents random turbulent motion, rather than infall motions or other systemic velocity shifts within the clump. The $\Delta v_{\mathrm{NT}}$ values for each clump are listed in Table 2.

\section{Appendix C Grain Alignment in $\mathbf{L 1 6 8 9}$}

In order to determine the degree of grain alignment in L1689, we applied the method described by Pattle et al. (2019) to our observations. We assume that the underlying relationship between $p$ and $I$ can be parameterized as

$$
p=p_{\sigma_{Q U}}\left(\frac{I}{\sigma_{Q U}}\right)^{-\alpha}
$$

where $p_{\sigma_{Q U}}$ is the polarization fraction at the rms noise level of the data $\sigma_{Q U}$, and $\alpha$ is a power-law index in the range
$0 \leqslant \alpha \leqslant 1$. We take $\sigma_{Q U}$ to be a directly measurable property of the data set, and aim to infer $p_{\sigma_{Q U}}$ and $\alpha$.

In order to determine $\alpha$ and $p_{\sigma_{Q U}}$ we fitted the relationship between $I$ and non-debiased observed polarization fraction $p^{\prime}$ with the mean of the Rice distribution, such that

$$
p^{\prime}(I)=\sqrt{\frac{\pi}{2}} \sigma_{p}(I) \mathcal{L}_{\frac{1}{2}}\left(-\frac{p(I)^{2}}{2 \sigma_{p}(I)^{2}}\right)
$$

where $\sigma_{p}$ is uncertainty on $p$ and $\mathcal{L}_{\frac{1}{2}}$ is a Laguerre polynomial of order $\frac{1}{2}$.

We took $p(I)$ to be as given in Equation (C1) and $\sigma_{p}(I) \approx \sigma_{Q U} / I$ (see Pattle et al. 2019 for a discussion of this approximation), and so Equation (C2) becomes

$$
p^{\prime}(I)=\sqrt{\frac{\pi}{2}}\left(\frac{I}{\sigma_{Q U}}\right)^{-1} \mathcal{L}_{\frac{1}{2}}\left(-\frac{p_{\sigma_{Q U}}^{2}}{2}\left(\frac{I}{\sigma_{Q U}}\right)^{2(1-\alpha)}\right) .
$$

We restricted each data set to the central region of 3 arcmin diameter over which exposure time is approximately constant and so rms noise is approximately flat (Friberg et al. 2016). We then estimated an rms noise value in our Stokes $Q$ and $U$ data,

$$
\sigma_{Q U}=\frac{1}{2 N} \sum_{i=1}^{N}\left(\sqrt{V_{Q, i}}+\sqrt{V_{U, i}}\right)
$$

where $N$ is the number of pixels in the central region of 3 arcmin diameter of the data set and $V_{Q}$ and $V_{U}$ are the variance values associated with each pixel, as determined in the data reduction process. Our measured values of $\sigma_{Q U}$ are listed in Table 4.

We then fitted each data set using Equation (C3). The results of this fitting are listed in Table 4 and shown in Figure 7 . We find that the fitting results for all regions agree with one another within error. We find $\alpha=0.55 \pm 0.03$ in L1689N, while we find $\alpha=0.59 \pm 0.06$ in SMM-16 and $\alpha=0.56 \pm 0.09$ in L1689B. This suggests that in both regions grains become less well aligned with the magnetic field as density increases, but that some degree of alignment persists at the highest densities that we observe.

In both IRAS 16293 and SMM-16 the reduced- $\chi^{2}$ values of the best-fitting models are significantly smaller than those of the null hypothesis behavior. The deviation of the data from the null hypothesis behavior can also be clearly seen in Figure 7. However, in both regions, and particularly in L1689N, the reduced- $\chi^{2}$ values of the best-fit models are greater than unity. This suggests that there is more variation in the $p-I$ relationship in L1689N than can be explained with this simple model alone.

In L1689B, which is significantly less bright than either L1689N or SMM-16, the reduced- $\chi^{2}$ value of the best-fitting model is somewhat smaller than that of the null hypothesis, but both are similar to unity. While Figure 7 shows a clear deviation from the null hypothesis behavior in L1689N and SMM-16, such a deviation is less apparent, although still somewhat visible, in L1689B. The level of grain alignment in L1689B is thus less well characterized than in either of the other regions, but the region shows behavior consistent with some degree of grain alignment being retained to high densities. 
Table 4

Results of Fitting Ricean-mean Model to the $p-I$ Relations of L1689N, SMM-16, and L1689B

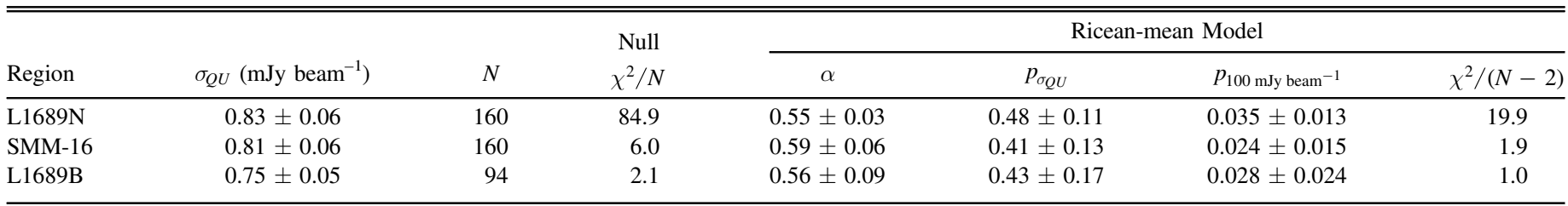

\section{ORCID iDs}

Kate Pattle (1) https://orcid.org/0000-0002-8557-3582 Shih-Ping Lai (i) https://orcid.org/0000-0001-5522-486X James Di Francesco iㅣ https://orcid.org/0000-0002-9289-2450 Sarah Sadavoy (1) https://orcid.org/0000-0001-7474-6874 Derek Ward-Thompson (i) https://orcid.org/0000-00031140-2761

Doug Johnstone (1) https://orcid.org/0000-0002-6773-459X Thiem Hoang (i) https://orcid.org/0000-0003-2017-0982 Doris Arzoumanian (다 https://orcid.org/0000-0002-1959-7201 Pierre Bastien (1) https://orcid.org/0000-0002-0794-3859 Tyler L. Bourke (1) https://orcid.org/0000-0001-7491-0048 Simon Coudé (i) https://orcid.org/0000-0002-0859-0805 Yasuo Doi (1) https://orcid.org/0000-0001-8746-6548 Chakali Eswaraiah (i) https://orcid.org/0000-0003-4761-6139 Lapo Fanciullo (1) https://orcid.org/0000-0001-9930-9240 Ray S. Furuya @ ittps://orcid.org/0000-0003-0646-8782 Jihye Hwang (i) https://orcid.org/0000-0001-7866-2686 Charles L. H. Hull (i) https://orcid.org/0000-0002-8975-7573 Jihyun Kang (i) https://orcid.org/0000-0001-7379-6263 Kee-Tae Kim (1) https://orcid.org/0000-0003-2412-7092 Florian Kirchschlager (ib https://orcid.org/0000-00023036-0184

Jungmi Kwon iㅏ https://orcid.org/0000-0003-2815-7774 Woojin Kwon (1) https://orcid.org/0000-0003-4022-4132 Chang Won Lee (이창원) 이 https://orcid.org/0000-00023179-6334

Tie Liu (刘铁) (10 https://orcid.org/0000-0002-5286-2564 Matt Redman (1) https://orcid.org/0000-0002-1021-9343 Archana Soam (ib https://orcid.org/0000-0002-6386-2906 Mehrnoosh Tahani 『 https://orcid.org/0000-0001-8749-1436 Motohide Tamura (1) https://orcid.org/0000-0002-6510-0681 Xindi Tang () https://orcid.org/0000-0002-4154-4309

\section{References}

Alves, F. O., Frau, P., Girart, J. M., et al. 2014, A\&A, 569, L1 Alves, F. O., Frau, P., Girart, J. M., et al. 2015, A\&A, 574, C4 Andersson, B.-G., Lazarian, A., \& Vaillancourt, J. E. 2015, ARA\&A, 53, 501 André, P., di Francesco, J., Ward-Thompson, D., et al. 2014, in Protostars and Planets VI, ed. H. Beuther et al. (Tucson, AZ: Univ. of Arizona Press), 27 André, P., Men'shchikov, A., Bontemps, S., et al. 2010, A\&A, 518, L102 Arzoumanian, D., André, P., Könyves, V., et al. 2019, A\&A, 621, A42 Astropy Collaboration, Price-Whelan, A. M., Hogg, D. W., et al. 2018, AJ, 156,123

Astropy Collaboration, Robitaille, T. P., Tollerud, E. J., et al. 2013, A\&A, 558, A33

Bacmann, A., García-García, E., \& Faure, A. 2016, A\&A, 588, L8

Bethell, T. J., Chepurnov, A., Lazarian, A., \& Kim, J. 2007, ApJ, 663, 1055 Caselli, P., Benson, P. J., Myers, P. C., \& Tafalla, M. 2002, ApJ, 572, 238

Chandler, C. J., Brogan, C. L., Shirley, Y. L., \& Loinard, L. 2005, ApJ, 632,371

Chandrasekhar, S., \& Fermi, E. 1953, ApJ, 118, 113

Chapin, E. L., Berry, D. S., Gibb, A. G., et al. 2013, MNRAS, 430, 2545

Chitsazzadeh, S., di Francesco, J., Schnee, S., et al. 2014, ApJ, 790, 129
Clarke, S. D., Whitworth, A. P., \& Hubber, D. A. 2016, MNRAS, 458, 319

Coudé, S., Bastien, P., Houde, M., et al. 2019, ApJ, 877, 88

Crapsi, A., Caselli, P., Walmsley, C. M., et al. 2005, ApJ, 619, 379

Crutcher, R. M., Nutter, D. J., Ward-Thompson, D., \& Kirk, J. M. 2004, ApJ, 600, 279

Crutcher, R. M., Wandelt, B., Heiles, C., Falgarone, E., \& Troland, T. H. 2010, ApJ, 725, 466

Currie, M. J., Berry, D. S., Jenness, T., et al. 2014, in ASP Conf. Ser., 485, Astronomical Data Analysis Software and Systems XXIII, ed. N. Manset \& P. Forshay (San Francisco, CA: ASP), 391

Davis, L. 1951, PhRv, 81, 890

Davis, L., Jr., \& Greenstein, J. L. 1951, ApJ, 114, 206

de Zeeuw, P. T., Hoogerwerf, R., de Bruijne, J. H. J., Brown, A. G. A., \& Blaauw, A. 1999, AJ, 117, 354

Dempsey, J. T., Friberg, P., Jenness, T., et al. 2013, MNRAS, 430, 2534

Doi, Y., Hasegawa, T., Furuya, R. S., et al. 2020, ApJ, 899, 28

Dolginov, A. Z., \& Mitrofanov, I. G. 1976, Ap\&SS, 43, 291

Draine, B. T. 2011, Physics of the Interstellar and Intergalactic Medium (Princeton, NJ: Princeton Univ. Press)

Draine, B. T., Dale, D. A., Bendo, G., et al. 2007, ApJ, 663, 866

Draine, B. T., \& Weingartner, J. C. 1996, ApJ, 470, 551

Enoch, M. L., Evans, N. J., II, Sargent, A. I., \& Glenn, J. 2009, ApJ, 692, 973

Fiedler, R. A., \& Mouschovias, T. C. 1993, ApJ, 415, 680

Friberg, P., Bastien, P., Berry, D., et al. 2016, Proc. SPIE, 9914, 991403

Friberg, P., Berry, D., Savini, G., et al. 2018, Proc. SPIE, 10708, 107083M

Girart, J. M., Rao, R., \& Marrone, D. P. 2006, Sci, 313, 812

Gómez, G. C., Vázquez-Semadeni, E., \& Zamora-Avilés, M. 2018, MNRAS, 480, 2939

Guillet, V., Girart, J. M., Maury, A. J., \& Alves, F. O. 2020, A\&A, 634, L15

Heiles, C., \& Troland, T. H. 2005, ApJ, 624, 773

Heitsch, F., Zweibel, E. G., mac Low, M.-M., Li, P., \& Norman, M. L. 2001, ApJ, 561, 800

Hennebelle, P., \& Inutsuka, S.-i. 2019, FrASS, 6, 5

Hildebrand, R. H. 1983, QJRAS, 24, 267

Hoang, T., \& Lazarian, A. 2008, MNRAS, 388, 117

Hull, C. L. H., Plambeck, R. L., Bolatto, A. D., et al. 2013, ApJ, 768, 159

Hull, C. L. H., \& Zhang, Q. 2019, FrASS, 6, 3

Jacobsen, S. K., Jørgensen, J. K., van der Wiel, M. H. D., et al. 2018, A\&A, 612, A72

Jessop, N. E., \& Ward-Thompson, D. 2000, MNRAS, 311, 63

Johnstone, D., Ciccone, S., Kirk, H., et al. 2017, ApJ, 836, 132

Jones, T. J., Bagley, M., Krejny, M., Andersson, B.-G., \& Bastien, P. 2015, AJ, 149,31

Jørgensen, J. K., van der Wiel, M. H. D., Coutens, A., et al. 2016, A\&A, 595, A117

Juvela, M., Ristorcelli, I., Marshall, D. J., et al. 2015, A\&A, 584, A93

Kataoka, A., Muto, T., Momose, M., et al. 2015, ApJ, 809, 78

Kim, S., Lee, C. W., Gopinathan, M., et al. 2020, ApJ, 891, 169

Kirchschlager, F., Bertrang, G. H. M., \& Flock, M. 2019, MNRAS, 488, 1211

Kirk, H., Dunham, M. M., Di Francesco, J., et al. 2017, ApJ, 838, 114

Kirk, J. M., Ward-Thompson, D., \& André, P. 2007, MNRAS, 375, 843

Kirk, J. M., Ward-Thompson, D., \& Crutcher, R. M. 2006, MNRAS, 369, 1445

Ko, C.-L., Liu, H. B., Lai, S.-P., et al. 2020, ApJ, 889, 172

Könyves, V., André, P., Men'shchikov, A., et al. 2010, A\&A, 518, L106

Krumholz, M. R., \& Federrath, C. 2019, FrASS, 6, 7

Kwon, J., Doi, Y., Tamura, M., et al. 2018, ApJ, 859, 4

Kwon, J., Tamura, M., Hough, J. H., et al. 2015, ApJS, 220, 17

Ladjelate, B., André, P., Könyves, V., et al. 2020, A\&A, 638, 74

Lazarian, A., \& Hoang, T. 2007, MNRAS, 378, 910

Lee, C. W., \& Myers, P. C. 2011, ApJ, 734, 60

Lee, C. W., Myers, P. C., \& Tafalla, M. 1999, ApJ, 526, 788

Lee, C. W., Myers, P. C., \& Tafalla, M. 2001, ApJS, 136, 703

Lee, H., Hoang, T., Le, N., \& Cho, J. 2020, ApJ, 896, 44

Lee, J.-E., Evans, N. J. I., Shirley, Y. L., \& Tatematsu, K. 2003, ApJ, 583, 789 
Li, H.-b., Fang, M., Henning, T., \& Kainulainen, J. 2013, MNRAS, 436, 3707

Li, H.-B., Jiang, H., Fan, X., Gu, Q., \& Zhang, Y. 2017, NatAs, 1, 0158

Liu, J., Qiu, K., Berry, D., et al. 2019, ApJ, 877, 43

Loren, R. B. 1989, ApJ, 338, 902

Loren, R. B., Wootten, A., \& Wilking, B. A. 1990, ApJ, 365, 269

Lynds, B. T. 1962, ApJS, 7, 1

Mairs, S., Johnstone, D., Kirk, H., et al. 2015, MNRAS, 454, 2557

Mairs, S., Lane, J., Johnstone, D., et al. 2017, ApJ, 843, 55

Mamajek, E. E. 2008, AN, 329, 10

Mathis, J. S., Mezger, P. G., \& Panagia, N. 1983, A\&A, 500, 259

Mathis, J. S., Rumpl, W., \& Nordsieck, K. H. 1977, ApJ, 217, 425

Mizuno, A., Fukui, Y., Iwata, T., Nozawa, S., \& Takano, T. 1990, ApJ, 356,184

Mouschovias, T. C., \& Spitzer, L. J. 1976, ApJ, 210, 326

Mundy, L. G., Wootten, A., Wilking, B. A., Blake, G. A., \& Sargent, A. I. 1992, ApJ, 385, 306

Nutter, D., Ward-Thompson, D., \& André, P. 2006, MNRAS, 368, 1833

Ortiz-León, G. N., Loinard, L., Dzib, S. A., et al. 2018, ApJL, 869, L33

Ostriker, E. C., Stone, J. M., \& Gammie, C. F. 2001, ApJ, 546, 980

Ostriker, J. 1964, ApJ, 140, 1056

Palmeirim, P., André, P., Kirk, J., et al. 2013, A\&A, 550, A38

Pan, Z., Li, D., Chang, Q., et al. 2017, ApJ, 836, 194

Pattle, K., \& Fissel, L. 2019, FrASS, 6, 15

Pattle, K., Lai, S.-P., Hasegawa, T., et al. 2019, ApJ, 880, 27

Pattle, K., Ward-Thompson, D., Berry, D., et al. 2017, ApJ, 846, 122

Pattle, K., Ward-Thompson, D., Kirk, J. M., et al. 2015, MNRAS, 450, 1094

Pineda, J. E., Maury, A. J., Fuller, G. A., et al. 2012, A\&A, 544, L7

Planck Collaboration, Ade, P. A. R., Aghanim, N., et al. 2015, A\&A, 576, A104

Planck Collaboration, Ade, P. A. R., Aghanim, N., et al. 2016, A\&A, 586, A138

Rao, R., Girart, J. M., Marrone, D. P., Lai, S.-P., \& Schnee, S. 2009, ApJ, 707, 921

Redman, M. P., Keto, E., Rawlings, J. M. C., \& Williams, D. A. 2004 MNRAS, 352, 1365

Redman, M. P., Rawlings, J. M. C., Nutter, D. J., Ward-Thompson, D., \& Williams, D. A. 2002, MNRAS, 337, L17

Ridge, N. A., di Francesco, J., Kirk, H., et al. 2006, AJ, 131, 2921

Roy, A., André, P., Palmeirim, P., et al. 2014, A\&A, 562, A138

Roy, A., Martin, P. G., Polychroni, D., et al. 2013, ApJ, 763, 55

Sadavoy, S. I., di Francesco, J., \& Johnstone, D. 2010, ApJL, 718, L32
Sadavoy, S. I., Myers, P. C., Stephens, I. W., et al. 2018, ApJ, 869, 115 Sadavoy, S. I., Stephens, I. W., Myers, P. C., et al. 2019, ApJS, 245, 2 Santos, F. P., Ade, P. A. R., Angilè, F. E., et al. 2017, ApJ, 837, 161

Santos, F. P., Chuss, D. T., Dowell, C. D., et al. 2019, ApJ, 882, 113

Schirmer, T., Abergel, A., Verstraete, L., et al. 2020, A\&A, 639, A144

Schlafly, E. F., \& Finkbeiner, D. P. 2011, ApJ, 737, 103

Schlegel, D. J., Finkbeiner, D. P., \& Davis, M. 1998, ApJ, 500, 525

Schnee, S., Brunetti, N., di Francesco, J., et al. 2013, ApJ, 777, 121

Seifried, D., Walch, S., Weis, M., et al. 2020, MNRAS, 497, 4196

Seo, Y. M., Hong, S. S., \& Shirley, Y. L. 2013, ApJ, 769, 50

Serkowski, K. 1962, AdA\&A, 1, 289

Soam, A., Liu, T., Andersson, B. G., et al. 2019, ApJ, 883, 95

Soam, A., Pattle, K., Ward-Thompson, D., et al. 2018, ApJ, 861, 65

Sohn, J., Lee, C. W., Park, Y.-S., et al. 2007, ApJ, 664, 928

Soler, J. D. 2019, A\&A, 629, A96

Soler, J. D., \& Hennebelle, P. 2017, A\&A, 607, A2

Soler, J. D., Hennebelle, P., Martin, P. G., et al. 2013, ApJ, 774, 128

Steinacker, J., Bacmann, A., Henning, T., \& Heigl, S. 2016, A\&A, 593, A6

Tafalla, M., Myers, P. C., Caselli, P., \& Walmsley, C. M. 2004, A\&A, 416, 191

Tang, Y.-W., Koch, P. M., Peretto, N., et al. 2019, ApJ, 878, 10

Vrba, F. J. 1977, AJ, 82, 198

Vrba, F. J., Strom, S. E., \& Strom, K. M. 1976, AJ, 81, 958

Walker, C. K., Lada, C. J., Young, E. T., \& Margulis, M. 1988, ApJ, 332, 335

Wang, J.-W., Lai, S.-P., Eswaraiah, C., et al. 2019, ApJ, 876, 42

Ward-Thompson, D., Kirk, J. M., Crutcher, R. M., et al. 2000, ApJL, 537, L135

Ward-Thompson, D., Pattle, K., Bastien, P., et al. 2017, ApJ, 842, 66

Ward-Thompson, D., Scott, P. F., Hills, R. E., \& André, P. 1994, MNRAS, 268, 276

Wenger, M., Ochsenbein, F., Egret, D., et al. 2000, A\&AS, 143, 9

Whittet, D. C. B., Hough, J. H., Lazarian, A., \& Hoang, T. 2008, ApJ, 674, 304

Wilking, B. A., Gagné, M., \& Allen, L. E. 2008, in Handbook of Star Forming Regions, Volume II: The Southern Sky, ed. B. Reipurth (San Francisco, CA: ASP), 351

Wootten, A. 1989, ApJ, 337, 858

Xu, X., Li, D., Dai, Y. S., Goldsmith, P. F., \& Fuller, G. A. 2020, ApJ, 898,122

Ysard, N., Abergel, A., Ristorcelli, I., et al. 2013, A\&A, 559, A133

Zhang, G. Y., Andre, P., Menshchikov, A., \& Wang, K. 2020, A\&A, 642, A76 